\title{
Presentations of finite simple groups: profinite and cohomological approaches
}

\author{
Robert Guralnick, William M. Kantor, Martin Kassabov \\ and Alexander Lubotzky
}

Dedicated to our friend and colleague Avinoam Mann

\begin{abstract}
We prove the following three closely related results:
(1) Every finite simple group $G$ has a profinite presentation with 2 generators and at most 18 relations.

(2) If $G$ is a finite simple group, $F$ a field and $M$ is an $F G$-module, then $\operatorname{dim} H^{2}(G, M) \leq$ (17.5) $\operatorname{dim} M$.

(3) If $G$ is a finite group, $F$ a field and $M$ is an irreducible faithful $F G$-module, then $\operatorname{dim} H^{2}(G, M) \leq(18.5) \operatorname{dim} M$.
\end{abstract}

Mathematics Subject Classification (2000). 20F05, 20J06, 20D05, 20 C20.

Keywords. Finite simple groups, generators, relations, presentations, profinite presentations, cohomology, second cohomology group.

\section{Contents}

1 Introduction . . . . . . . . . . . . . . . . . . . . . . 470

2 General strategy and notation . . . . . . . . . . . . . . 473

3 Preliminaries on cohomology . . . . . . . . . . . . . . . . . . . . 474

4 Covering groups . . . . . . . . . . . . . . . . . 480

5 Faithful irreducible modules and Theorem C . . . . . . . . . . . 482

The authors were partially supported by NSF grants DMS 0140578, DMS 0242983, DMS 0600244 and DMS 0354731. The authors are grateful for the support and hospitality of the Institute for Advanced Study, where this research was carried out. The research by the fourth author also was supported by the ISF, the Ambrose Monell Foundation and the Ellentuck Fund. We also thank the two referees for their careful reading and comments. 


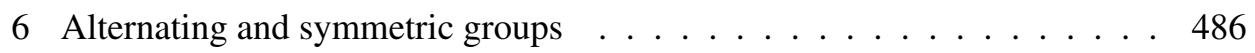

$6.1 p>3 \ldots \ldots \ldots \ldots \ldots \ldots \ldots \ldots \ldots \ldots$

$6.2 p=3 \ldots \ldots \ldots \ldots \ldots \ldots \ldots \ldots \ldots \ldots \ldots$

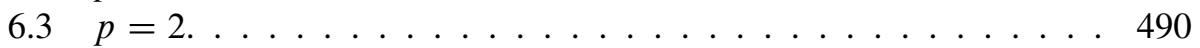

7 SL: low rank . . . . . . . . . . . . . . . . . . . . . . 493

$7.1 \quad \mathrm{SL}(2) \ldots \ldots \ldots \ldots \ldots \ldots \ldots$

$7.2 \quad \mathrm{SL}(3) \ldots \ldots \ldots \ldots \ldots \ldots$

$7.3 \mathrm{SL}(4) \ldots \ldots \ldots \ldots \ldots \ldots \ldots$

8 SL: the general case . . . . . . . . . . . . . . . . . . . . 501

9 Low rank groups . . . . . . . . . . . . . . . . . . . . . . . . . 504

10 Groups of Lie type - the general case . . . . . . . . . . . . . . 511

11 Sporadic groups . . . . . . . . . . . . . . . . . . . 512

12 Higher cohomology . . . . . . . . . . . . . . . . . . . . 513

13 Profinite versus discrete presentations $\ldots \ldots \ldots \ldots$

\section{Introduction}

The main goal of this paper is to prove the following three results which are essentially equivalent to each other. Recall that a quasisimple group is one that is perfect and simple modulo its center. Note that the last theorem is about all finite groups.

Theorem A. Every finite quasisimple group $G$ has a profinite presentation with 2 generators and at most 18 relations.

Theorem B. If $G$ is a finite quasisimple group, $F$ a field and $M$ an $F G$-module, then $\operatorname{dim} H^{2}(G, M) \leq(17.5) \operatorname{dim} M$.

Theorem C. If $G$ is a finite group, $F$ a field and $M$ an irreducible faithful $F G$-module, then $\operatorname{dim} H^{2}(G, M) \leq(18.5) \operatorname{dim} M$.

All three theorems depend on the classification of finite simple groups. One could prove Theorems A and B independently of the classification for the known simple groups.

We abuse notation somewhat and say that an $F G$-module is faithful if $G$ acts faithfully on $M$. We call $M$ a trivial $G$-module if it is 1-dimensional and $G$ acts trivially on $M$.

In [21], the predecessor of this article, we showed that every finite non-abelian simple group, with possible exception of the family ${ }^{2} G_{2}\left(3^{2 k+1}\right)$, has a bounded short presentation (with at most 1000 relations - short being defined in terms of the sums of the lengths of the relations). We deduced results similar to the first two theorems above but with larger constants. In [22], we show that every finite simple group (with 
possible exception of $\left.{ }^{2} G_{2}\left(3^{2 k+1}\right)\right)$ has a presentation with 2 generators and at most 100 relations.

In many cases, the results proved here and in [22] are much better, e.g., for $A_{n}$ and $S_{n}$, we produce presentations with 3 generators and 7 relations [22]. Here we give still better results for these groups in the profinite case - there are profinite presentations with 2 generators and 4 relations.

We believe that with more effort (and some additional ideas) the constants in these three theorems may be dropped to 4,2 and $1 / 2$ respectively.

One of the methods used in this paper is possibly of as much interest as the results themselves. We show how to combine cohomological and profinite presentations arguments - by going back and forth between the two topics to deduce results on both. The bridge between the two subjects is a formula given in [34] which states: If $G$ is a finite group and $\hat{r}(G)$ is the minimal number of relations in a profinite presentation of $G$, then

$$
\hat{r}(G)=\sup _{p} \sup _{M}\left(\left\lceil\frac{\operatorname{dim} H^{2}(G, M)-\operatorname{dim} H^{1}(G, M)}{\operatorname{dim} M}\right\rceil+d(G)-\xi_{M}\right),
$$

where $d(G)$ is the minimum number of generators for $G, p$ runs over all primes, $M$ runs over all irreducible $\mathbb{F}_{p} G$-modules, and $\xi_{M}=0$ if $M$ is the trivial module and 1 if not. By [19], if $G$ is a quasisimple finite group, then for every $\mathbb{F}_{p} G$ module $M$,

$$
\operatorname{dim} H^{1}(G, M) \leq(1 / 2) \operatorname{dim} M .
$$

Set

$$
h_{p}^{\prime}(G)=\max _{M} \frac{\operatorname{dim} H^{2}(G, M)}{\operatorname{dim} M}, \quad \text { and } \quad h^{\prime}(G)=\max _{p} h_{p}^{\prime}(G),
$$

where $M$ ranges over nontrivial irreducible $\mathbb{F}_{p} G$-modules. If $G$ is a finite quasisimple group, then $d(G) \leq 2\left[4\right.$, Theorem B] and $\operatorname{dim} H^{2}\left(G, \mathbb{F}_{p}\right) \leq 2[16$, pp. 312-313] $)$ and so

$$
\max \left\{2,\left\lceil h^{\prime}(G)+1 / 2\right\rceil\right\} \leq \hat{r}(G) \leq \max \left\{4,\left\lceil h^{\prime}(G)+1\right\rceil\right\} .
$$

This explains how Theorems A and B are related and are essentially equivalent. We see in Section 5 that Theorem B implies Theorem C. On the other hand, the bound for Schur multipliers for finite simple groups and Theorem $\mathrm{C}$ implies a version of Theorem B.

We also define

$$
h(G)=\max _{M, p} \frac{\operatorname{dim} H^{2}(G, M)}{\operatorname{dim} M},
$$

where $M$ ranges over all $\mathbb{F}_{p} G$-modules.

We now give an outline of the paper. After some preparation in Sections 3, 4, and 5, we show in Sections 6, 7 and 9, respectively, that: 
Theorem D. For every $n, h\left(A_{n}\right)<3$ and $h\left(S_{n}\right)<3$ and $\hat{r}\left(A_{n}\right)$ and $\hat{r}\left(S_{n}\right) \leq 4$.

Theorem E. For every prime power $q$ and $2 \leq n \leq 4, h(\operatorname{SL}(n, q)) \leq 2$.

Theorem F. $\max \{h(G), \hat{r}(G)\} \leq 6$ for each rank 2 quasisimple finite group $G$ of Lie type.

In fact, the results are more precise - see Sections 6, 7 and 9 for details.

From (1.4) we see that Theorems D, E and F imply that all the groups in those theorems have profinite presentations with a small number of relations. In Sections 8 and 10, we repeat our "gluing" arguments from [21, §6.2] to show how to deduce from these cases the existence of bounded (profinite) presentations for all the quasisimple finite groups of Lie type. In fact, this time the proof is easier and the result is stronger as we do not insist of having a short presentation as we did in [21]; we count only the number of relations but not their length. Moreover, Lemma 3.15 gives an interesting method for saving relations which seems to be new (the analog is unlikely to work for discrete presentations). In Section 11, we discuss the sporadic simple groups. If a Sylow $p$-subgroup has order at most $p^{m}$, one can use the main result of [30] to deduce the bound $h_{p}^{\prime}(G) \leq 2 \mathrm{~m}$. In many sporadic cases, discrete presentations for the groups are known [51] and the results follow. There are not too many additional cases to consider.

This completes the outline of the proof of Theorem A. Applying (1.4) in the reverse direction we deduce Theorem $\mathrm{B}$ (at least for $\mathbb{F}_{p}$ - however, changing the base field does not change the ratio $\operatorname{dim} H^{2}(G, M) / \operatorname{dim} M$ - see Lemma 3.2 and the discussion following it). In Section 5, we prove Theorem 5.3 which shows that Theorem B implies Theorem C.

Holt [30] conjectured Theorem $\mathrm{C}$ for some constant $C$. He proved that

$$
\operatorname{dim} H^{2}(G, M) \leq 2 e_{p}(G) \operatorname{dim} M,
$$

for $M$ an irreducible faithful $G$-module, where $p^{e_{p}(G)}$ is the order of a Sylow $p$-subgroup of $G$. Holt also reduced his proof to simple groups. However, he was proving a weaker result than we are aiming for, and his reduction methods are not sufficient for our purposes.

As we have already noted in (1.2), the analog of Theorem B for $H^{1}$ holds with constant $1 / 2$. It is relatively easy to see that this implies that the analog of Theorem $\mathrm{C}$ for $H^{1}$ with constant $1 / 2$ is valid. We give examples to show that the situation for higher cohomology groups is different (see Section 12). In particular, the following holds:

Theorem G. Let $F$ be an algebraically closed field of characteristic $p>0$ and let $k$ be a positive integer. 
There exists a sequence of finite groups $G_{i}, i \in \mathbb{N}$ and irreducible faithful $F G_{i}$ modules $M_{i}$ such that:

(1) $\lim _{i \rightarrow \infty} \operatorname{dim} M_{i}=\infty$,

(2) $\operatorname{dim} H^{k}\left(G_{i}, M_{i}\right) \geq e\left(\operatorname{dim} M_{i}\right)^{k-1}$ for some constant $e=e(k, p)>0$, and

(3) if $k \geq 3$, then $\lim _{i \rightarrow \infty} \frac{\operatorname{dim} H^{k}\left(G_{i}, M_{i}\right)}{\operatorname{dim} M_{i}}=\infty$.

Thus the analog of Theorem $\mathrm{C}$ for $H^{k}$ with $k \geq 3$ does not hold for any constant although it is still possible that an analog of Theorem B holds. This also shows that $\operatorname{dim} H^{2}(G, M)$ can be arbitrarily large for faithful absolutely irreducible modules it is not known whether this is possible for $H^{1}(G, M)$ under the same hypotheses. We suspect that there is an upper bound for $\operatorname{dim} H^{k}(G, M)$ of the same form as the lower bound in (2) above.

Finally, in Section 13 we give some applications of the results in [21] and the current paper for general finite groups, as well as some questions. An especially intriguing question is related to the fact that $\hat{r}(G) \leq r(G)$, the minimal number of relations required in any presentation of the group $G$. As far as we know, it is still not known whether for some finite group $G$, we can have $\hat{r}(G)<r(G)$.

There is a long history of studying presentations of groups and, in particular, the number and length of relations required for finite groups. Presentations of groups also rise in connection with various problems about counting isomorphism classes of groups. Much of the work done recently on these questions (e.g., [21], [31], [33], and [36, Chapter 2]) was motivated by the paper [39] of Avinoam Mann. We dedicate this paper to him on the occasion of his retirement.

\section{General strategy and notation}

We outline a method in order to obtain bounds of the form $\operatorname{dim} H^{2}(G, M) \leq C \operatorname{dim} M$ for some constant $C$. Here $G$ is a finite group and $M$ is an $F G$-module with $F$ a field of characteristic $p>0$ (in characteristic zero, $H^{2}(G, M)=0$ - see Corollary 3.12). There are several techniques that we use to reduce the problem to smaller groups.

The first is to use the long exact sequence for cohomology (Lemma 3.3) to reduce to the case that $M$ is irreducible. Then we use Lemma 3.2, which allows us to assume that we are over an algebraically closed field and that $M$ is absolutely irreducible (occasionally, it is convenient to use this in the reverse direction and assume that $M$ is finite and over $\mathbb{F}_{p}$ - see the discussion after Lemma 3.2). We also use the standard fact that $H^{2}(G, M)$ embeds in $H^{2}(H, M)$ whenever $H \leq G$ contains a Sylow $p$-subgroup of $G$ [17, p. 91]. Typically, $M$ will no longer be irreducible as an $F H$-module, but we can reduce to that case as above.

We use these reductions often without comment. 
We use our results on low rank finite groups of Lie type and the alternating groups to provide profinite presentations for the larger rank finite groups of Lie type, and so also bounds for $H^{2}$ via (1.4).

We use standard terminology for finite groups. In particular, $\mathrm{F}(G)$ is the Fitting subgroup and $O_{p}(G)$ is the maximal normal $p$-subgroup of $G$. A component is a subnormal quasisimple subgroup of $G$. The (central) product of all components of $G$ is denoted by $\mathrm{E}(G)$. Note that $\mathrm{E}(G)$ and $F(G)$ commute. The generalized Fitting subgroup is $\mathrm{F}^{*}(G):=\mathrm{E}(G) \mathrm{F}(G)$. We let $C_{t}$ denote the cyclic group of order $t$. See [2] for a general reference for finite group theory. We also use [16] as a general reference for properties of the finite simple groups - the Schur multipliers and outer automorphism groups of all the simple groups are given there.

If $M$ is an $H$-module, $M^{H}$ is the set of $H$ fixed points on $M$ and $[H, M]$ is the submodule generated by $\{h v-v \mid h \in H, v \in M\}$. Note that $[H, M]$ is the smallest submodule $L$ of $M$ such that $H$ acts trivially on $M / L$. If $V$ is a module for the subgroup $H$ of $G, V_{H}^{G}$ is the induced module.

\section{Preliminaries on cohomology}

Most of the results in this section are well known. See [6], [10], [37] and [17] for standard facts about group cohomology.

We first state a result that is an easy corollary of Wedderburn's theorem on finite division rings. We give a somewhat different proof based on Lang's theorem (of course, Wedderburn's theorem is a special case of Lang's Theorem). See also a result of Brauer $[15,19.3]$ that is slightly weaker.

Lemma 3.1. Let $K$ be a (possibly infinite) field of characteristic $p>0$, and let $G$ be a finite group. Let $V$ be an irreducible KG-module.

(1) There is a finite subfield $F$ of $K$ and an irreducible $F G$-module $W$ with $V \cong$ $W \otimes_{F} K$.

(2) $\operatorname{End}_{K G}(V)$ is a field.

Proof. Clearly, (1) implies (2) by Wedderburn's Theorem and Schur's Lemma. One can give a more direct proof. Let $F$ be a finite subfield of $K$. Then $B:=K G \cong$ $F G \otimes_{F} K$. Thus, $B / \operatorname{Rad}(B)$ is a homomorphic image of $(F G / \operatorname{Rad}(F G)) \otimes_{F} K$. By Wedderburn's Theorem, $F G / \operatorname{Rad}(F G)$ is a direct product of matrix rings over fields, and so the same is true for $B / \operatorname{Rad}(B)$. Thus, $B / \operatorname{Ann}_{B}(V) \cong M_{S}\left(K^{\prime}\right)$ for some extension field $K^{\prime} / K$. Since $K^{\prime} \cong \operatorname{End}_{G}(V)$, (2) follows.

We now prove (1). Set $n=\operatorname{dim} V$. Let $\phi: G \rightarrow \operatorname{GL}(n, K)$ be the representation determined by $V$. Let $F$ be the subfield of $K$ generated by the traces of elements of $\phi(g) \in G$ acting on $V$. Let $q$ denote the cardinality of the finite field $F$. 
Let $\sigma$ denote the $q$ th power map. Note that $F$ is the fixed field of $\sigma$. Then $\sigma$ induces an endomorphisms of $K G$ and so a twisted version of $V$, which we denote by $V^{\prime}$. Let $L$ denote the algebraic closure of $K$. Since the character of $V$ is defined over $F$, it follows that the characters of $V$ and $V^{\prime}$ are equal (and indeed, similarly for the Brauer characters). This implies that $V^{\prime} \cong V$ as $K G$-modules (or equivalently as $L G$-modules). Thus, there exist $U \in \mathrm{GL}(n, K)$ with $U \phi(g) U^{-1}=\sigma(\phi(g))$ for all $g \in G$. By Lang's theorem, $U=X^{-\sigma} X$ for some invertible matrix $X$ (over $L$ ). This implies that $\phi^{\prime}(g):=X \phi(g) X^{-1}=\sigma\left(X \phi(g) X^{-1}\right)$ defines a representation from $G$ into $\mathrm{GL}(n, F)$. Let $U$ be the corresponding module. Clearly, $V \cong U \otimes_{F} K$.

We state another result about extensions of scalars.

Lemma 3.2. Let $G$ be a finite group and $F$ a field. Let $M$ be an $F G$-module.

(1) If $K$ is an extension field of $F$, then $H^{2}(G, M) \otimes_{F} K$ and $H^{2}\left(G, M \otimes_{F} K\right)$ are naturally isomorphic, and in particular have the same dimension.

(2) If $M$ is irreducible and $F$ has positive characteristic, then $E:=\operatorname{End}_{G}(M)$ is a field, $M$ is an absolutely irreducible $E G$-module and $\operatorname{dim}_{F} H^{2}(G, M)=$ $[E: F] \operatorname{dim}_{E} H^{2}(G, M)$.

Proof. These results are well known. See [10, 0.8] for the first statement. By Lemma 3.1, $E$ is a field. Clearly, $M$ is an absolutely irreducible $E G$-module, and so $H^{2}(G, M)$ is also a vector space over $E$. The last equality holds for any finite dimensional vector space over $E$.

The previous result allows us to change fields in either direction. If $F$ is algebraically closed of characteristic $p>0$ and $M$ is an irreducible $F G$-module, then $M$ is defined over some finite field $E$-i.e. there is an absolutely irreducible $E G$-module $V$ such that $M=V \otimes_{E} F$ and we can compute the relevant ratios of dimensions over either field. Similarly, if $M$ is an irreducible $F G$-module with $F$ a finite field, then we can view $M$ an $E G$-module, where $E=\operatorname{End}_{G}(V)$, and so assume that $M$ is absolutely irreducible. Alternatively, we can view $M$ as an $\mathbb{F}_{p} G$-module.

See [10, III.6.1 and III.6.2] for the next two results.

Lemma 3.3. Let $G$ be a group and $0 \rightarrow X \rightarrow Y \rightarrow Z \rightarrow 0$ a short exact sequence of $G$-modules. This induces an exact sequence:

$$
\begin{aligned}
& 0 \rightarrow H^{0}(G, X) \quad \rightarrow H^{0}(G, Y) \rightarrow H^{0}(G, Z) \rightarrow H^{1}(G, X) \rightarrow \cdots \\
& \rightarrow H^{j-1}(G, Z) \rightarrow H^{j}(G, X) \rightarrow H^{j}(G, Y) \rightarrow H^{j}(G, Z) \rightarrow \cdots
\end{aligned}
$$

In particular, $\operatorname{dim} H^{j}(G, Y) \leq \operatorname{dim} H^{j}(G, X)+\operatorname{dim} H^{j}(G, Z)$ for any integer $j \geq 0$. 
Lemma 3.4 (Shapiro's Lemma). Let $G$ be a finite group and $H$ a subgroup of $G$. Let $V$ be an FH-module. Then $H^{j}(H, V) \cong H^{j}\left(G, V_{H}^{G}\right)$ for any integer $j \geq 0$.

Lemma 3.5. Let $G$ have a cyclic Sylow p-subgroup. Let $F$ be a field of characteristic $p$. If $M$ is an indecomposable $F G$-module and $j$ is a non-negative integer, then $\operatorname{dim} H^{j}(G, M) \leq 1$.

Proof. By a result of D. G. Higman (see [8, 3.6.4]), $M$ is a direct summand of $W_{P}^{G}$, where $P$ is a Sylow $p$-subgroup of $G$ and $W$ is an $F P$-module. Since $M$ is indecomposable, we may assume that $W$ is an indecomposable $P$-module. By Shapiro's Lemma (Lemma 3.4), $H^{j}(G, M)$ is a summand of $H^{j}(P, W)$. So it suffices to assume that $G=P$ is a cyclic $p$-group and $W$ is an indecomposable $P$-module (which is equivalent to saying $W$ is a cyclic $F P$-module).

In this case we show that $\operatorname{dim} H^{j}(P, W)=1$ unless $W$ is free (in which case the dimension is 0 ) by induction on $j$. If $j=0$, this is clear. So assume that $W$ is not free. Since $W$ is self cyclic and self dual, it embeds in a rank one free module $V$. Then $H^{i}(P, V)=0$ and by Lemma 3.3, $H^{i}(P, W) \cong H^{i-1}(P, V / W)$ and so is 1-dimensional (since $V / W$ is nonzero and cyclic).

The next result is standard - cf. [17, p. 91].

Lemma 3.6. If $H$ contains a Sylow p-subgroup of $G$, then the restriction map $H^{i}(G, M) \rightarrow H^{i}(H, M)$ is an injection.

The next result is an easy consequence of the Hochschild-Serre spectral sequence [37, p. 337]. See also [29].

Lemma 3.7. Let $N$ be a normal subgroup of $G, F$ a field and $M$ an $F G$-module. Then $\operatorname{dim} H^{q}(G, M) \leq \sum_{i+j=q} \operatorname{dim} H^{i}\left(G / N, H^{j}(N, M)\right)$.

We single out the previous lemma for the cases $q=1$,2. See [37, pp. 354-355] or [30, Lemma 2.1].

Lemma 3.8. Let $N$ be a normal subgroup of $H$ and let $M$ be an $F H$-module. Then

(1) $\operatorname{dim} H^{1}(H, M) \leq \operatorname{dim} H^{1}\left(H / N, M^{N}\right)+\operatorname{dim} H^{1}(N, M)^{H}$, and

(2) $\operatorname{dim} H^{2}(H, M) \leq \operatorname{dim} H^{2}\left(H / N, M^{N}\right)+\operatorname{dim} H^{2}(N, M)^{H}$

$$
+\operatorname{dim} H^{1}\left(H / N, H^{1}(N, M)\right) \text {. }
$$

We shall use the following well-known statements without comment.

Lemma 3.9. If $G$ is perfect, then $H^{1}\left(G, \mathbb{F}_{p}\right)=0$ and $\operatorname{dim} H^{2}\left(G, \mathbb{F}_{p}\right)$ is the p-rank of the Schur multiplier of $G$. 
We also use the following consequence of the Künneth formula.

Lemma 3.10. Let $F$ be a field and let $H=H_{1} \times \cdots \times H_{t}$ with the $H_{i}$ finite groups. Let $M_{i}$ be an irreducible $F H_{i}$-module for each $i$ and set $M=\bigotimes_{i=1}^{t} M_{i}$, an irreducible FH-module. Then

(1) $H^{r}(H, M)=\bigoplus_{\left(e_{i}\right)} H^{e_{1}}\left(H_{1}, M_{1}\right) \otimes \cdots \otimes H^{e_{t}}\left(H_{t}, M_{t}\right)$, where the sum is over all $\left(e_{i}\right)$ with the $e_{i}$ non-negative integers and $\sum e_{i}=r$.

(2) If $H_{i}$ acts nontrivially on $M_{i}$ for each $i$, then $H^{r}(H, M)=0$ for $r<t$ and $\operatorname{dim} H^{t}(H, M)=\prod \operatorname{dim} H^{1}\left(H_{i}, M_{i}\right)$.

(3) If each $H_{i}$ is quasisimple and each $M_{i}$ is nontrivial, then $\operatorname{dim} H^{t}(H, M) \leq$ $\operatorname{dim} M / 2^{t}$.

(4) If the $H_{j}$ are perfect for $j>1, M_{1}$ is nontrivial and $M_{j}$ is trivial for $j>1$, then $H^{2}(H, M) \cong H^{2}\left(H_{1}, M_{1}\right)$.

Proof. The first statement is just the Künneth formula as given in [8, 3.5.6], and the second statement follows immediately since $H^{0}\left(H_{i}, M_{i}\right)=0$. If $H_{i}$ is quasisimple, then (2) and (1.2) imply (3). Finally (4) follows from (1) and the fact that, by Lemma 3.9, $H^{1}\left(H_{j}, M_{j}\right)=0$ for $j>1$.

Note that there are quite a number of terms involved in $H^{r}(H, M)$ in the lemma above. Fortunately, when $r$ is relatively small, most terms will be 0 .

See $[6,35.6]$ for the next result.

Lemma 3.11. Assume that $N$ is normal in $H$ and $H^{r-1}(N, M)=0$. Then there is an exact sequence

$$
0 \rightarrow H^{r}\left(H / N, M^{N}\right) \rightarrow H^{r}(H, M) \rightarrow H^{r}(N, M)^{H} .
$$

We single out a special case of the previous lemma.

Corollary 3.12. Let $H$ be a finite group with a normal subgroup $N$. Let $M$ be an $\mathrm{FH}$-module.

(1) If $M^{N}=H^{j-1}(N, M)=0$, then the restriction map $H^{j}(H, M) \rightarrow H^{j}(N, M)$ is injective.

(2) If $N$ has order that is not a multiple of the characteristic of $M$ and $M^{N}=0$, then $H^{j}(H, M)=0$ for all $j$.

(3) If $N$ has order that is not a multiple of the characteristic of $M$ and $M^{N}=M$, then $H^{j}(H / N, M) \cong H^{j}(H, M)$ for all $j$. 
Proof. (1) is an immediate consequence of the previous lemma. Under the assumptions of (2), $M$ is a projective $F N$-module and so $H^{j}(N, M)=0$ for all $j>0$ and $H^{0}(N, M)=0$ by hypothesis. Thus (2) follows by induction on $j$ and (1). Note that (3) is a special case of Lemma 3.11.

The previous corollary in particular illustrates the well-known result that all higher cohomology groups for finite groups vanish in characteristic 0 . So we will always assume our fields have positive characteristic in what follows.

It is also convenient to mention a special case of Lemma 3.11 for $H^{1}$.

Lemma 3.13. Let $G$ be a finite group with $p$ a prime. Let $N$ be a normal $p$-subgroup of $G$ and $V$ an $\mathbb{F}_{p} G$-module with $N$ acting trivially on $V$. Then $\operatorname{dim} H^{1}(G, V) \leq$ $\operatorname{dim} H^{1}(G / N, V)+\operatorname{dim} \operatorname{Hom}_{G}(N, V)$.

Lemma 3.14. Let $A$ and $B$ be quasisimple groups with trivial Schur multipliers, and let $G=A \times B$. Then $d(G)=2$ and $\hat{r}(G)=\max \{\hat{r}(A), \hat{r}(B)\}$.

Proof. Since $d(A)=d(B)=2$, it follows that $d(G)=2$ unless possibly $A \cong B$. In that case $d(G)=2$ follows from the fact that the set of generating pairs of a finite simple group are not a single orbit under the automorphism group (e.g., use the main result of [20]). The last statement now follows by Lemma 3.10 and (1.1).

Since (1.1) does not give an explicit presentation, we cannot give one in the previous result. It would be interesting to do so.

The next result is an interesting way of giving profinite presentations with fewer relations than one might expect by giving presentations with more generators than the minimum required. Recall that a profinite presentation for a finite group $G$ is a free profinite group $F$ and a finite subset $U$ of $F$ such that if $R$ is the closed normal subgroup generated by $U, G \cong F / R$.

We show in the next result that if $G$ has a profinite presentation with $d(G)+c$ generations and $e$ relations, then it has a profinite presentation with $d(G)$ generators and $e-c$ relations. Often, we will give profinite presentations with more than the minimum number of generators required and so we deduce the existence of another profinite presentation with $d(G)$ generators and fewer relations. We do not know how to make this explicit and if this is true for discrete presentations. Indeed, the best result we know is that if $G$ has a (discrete) presentation with $r$ relations, then it is has a (discrete) presentation with $d(G)$ generators and $r+d(G)$ relations (see [21, Lemma 2.1]).

If $M$ is an $\mathbb{F}_{p} G$-module, let $d_{G}(M)$ be the minimum size of a generating set for $M$ as an $\mathbb{F}_{p} G$-module. The key result is in [34, Theorem 0.2], which asserts that if $G=F / R$ is a finite group, $F$ is a free profinite group and $R$ is a closed normal subgroup of $F$, then the minimal number of elements needed to generate $R$ as a closed normal subgroup of $F$ is equal to $\max _{p}\left\{d_{G}(M(p)\}\right.$, where $M(p)$ is the $G$-module 
$R /[R, R] R^{p}$ and $p$ ranges over all primes. Moreover, by $[17,2.4]$ the structure of $M(p)$ depends only on the rank of $F$.

Lemma 3.15. Let $G$ be a finite group. Consider a profinite presentation $G=F / R$ where $F$ is the free profinite group on $d(G)+c$ generators. Let $e$ to be the minimal number of elements required to generate $R$ as a closed normal subgroup of $F$. Then $\hat{r}(G)=e-c$. In particular, the minimal number of relations occurs when the number of generators is minimal, and only in that case.

Proof. Set $M=R /[R, R]$ and $M(p)=M / p M$ for $p$ a prime. So $M$ is the relation module for $G$ in this presentation and $M(p)$ is an $\mathbb{F}_{p} G$-module.

As noted above, $R$ is normally generated (as a closed subgroup) in $F$ by $e$ elements, where $e=\max _{p}\left\{d_{G}(M(p)\}\right.$. Also as noted above the structure of $M(p)$ only depends on the number of generators for $F$ and not on the particular presentation. So we may assume that all but $d(G)$ generators in the presentation are sent to 1 , whence we see that $M(p)=N(p) \oplus X_{p}$ where $X_{p}$ is a free $\mathbb{F}_{p} G$-module of rank $c$ and $N(p)$ is the $p$-quotient of the relation module for a minimal presentation.

Now the first statement follows from the elementary fact that, if an $\mathbb{F}_{p} G$-module $Y$ can be generated by $s$ elements but no fewer, then the $\mathbb{F}_{p} G$-module $Y \oplus \mathbb{F}_{p} G$ is generated by $s+1$ elements but no fewer. Indeed, this holds for any finite dimensional algebra $A$ over a field - for by Nakayama's Lemma, we may assume that $A$ is semisimple and so reduce to the case that $A$ is a simple algebra, where the result is clear.

The last statement is now an immediate consequence.

Lemma 3.16. Let $G$ be a finite group with a normal abelian p-subgroup L. Let $V$ be an irreducible $\mathbb{F}_{p} G$-module.

(1) There is an exact sequence of $G$-modules,

$$
0 \rightarrow \operatorname{Ext}_{\mathbb{Z}}(L, V) \rightarrow H^{2}(L, V) \rightarrow \wedge^{2}\left(L^{*}\right) \otimes V \rightarrow 0
$$

(2) $\operatorname{dim} H^{2}(L, V)^{G} \leq \operatorname{dim}\left((L / p L)^{*} \otimes V\right)^{G}+\operatorname{dim}_{F}\left(\wedge^{2}(L / p L)^{*} \otimes V\right)^{G}$.

(3) If $G=L$, then $\operatorname{dim} H^{2}\left(G, \mathbb{F}_{p}\right)=d(d+1) / 2$ where $d=d(G)$.

Proof. Since $G$ acts irreducibly on $V$, it follows that $L$ acts trivially on $V$.

It is well known (cf. [10, p. 127] or [7]) that when $L$ is abelian and acts trivially on $V$, there is a (split) short exact sequence as in (1) in the category of abelian groups. Here $\operatorname{Ext}_{\mathbb{Z}}(L, V)$ is the subspace of $H^{2}(L, V)$ corresponding to abelian extensions of $L$ by $V$. The natural maps are $G$-equivariant, giving (1). Note that $\operatorname{Ext}_{\mathbb{Z}}(L, V) \cong \operatorname{Hom}(L / p L, V) \cong(L / p L)^{*} \otimes V$ even as $G$-modules. Also, $\wedge^{2}\left(L^{*}\right) \otimes V \cong \wedge^{2}(L / p L)^{*} \otimes V$ since $V$ is elementary abelian.

Taking $G$-fixed points gives (2), and taking $G=L$ and $V=\mathbb{F}_{p}$ gives (3). 
Lemma 3.17. Let $T$ be a finite cyclic group of order $(q-1) / d$ acting faithfully on the irreducible $\mathbb{F}_{p} T$-module $X$ of order $q=p^{e}$. Set $Y=\wedge^{2}(X)$. Assume either that $d<p$ or that both $d=3$ and $q>4$. Then

(1) $Y$ is multiplicity free as a $T$-module; and

(2) $X$ is not isomorphic to a submodule of $Y$.

Proof. Let $x \in T$ be a generator. Thus, $x$ acts on $V$ with eigenvalue $\lambda \in \mathbb{F}_{q}$ of order $(q-1) / d$. It is straightforward to see that $Y \otimes_{\mathbb{F}_{p}} \mathbb{F}_{q}$ is a direct sum of submodules on which $x$ acts via $\lambda^{\left(p^{i}+p^{j}\right)}$ where $1 \leq i<j<e$. These submodules are all nonisomorphic (if not, then $d\left(p^{i}+p^{j}\right) \equiv d\left(p^{i^{\prime}}+p^{j^{\prime}}\right)$ modulo $p^{e}-1$ for some distinct pairs $\{i, j\}$ and $\left.\left\{i^{\prime}, j^{\prime}\right\}\right)$ and similarly are not isomorphic to $X$.

Lemma 3.18. Let $G$ be a finite group. Let $V$ be an irreducible $\mathbb{F}_{p} G$-module of dimension e. Then $\wedge^{2}(V)$ can be generated by $e-1$ elements as an $\mathbb{F}_{p} G$-module. In particular, $\operatorname{dim} \operatorname{Hom}_{G}\left(\wedge^{2}(V), W\right) \leq(e-1) \operatorname{dim} W$ for any $\mathbb{F}_{p} G$-module $W$.

Proof. Choose a basis $v=v_{1}, \ldots, v_{e}$ for $V$. It is clear that $v_{1} \wedge v_{j}, 2 \leq j \leq e$, is a generating set for $\wedge^{2}(V)$ as a $G$-module, which proves the first statement. The second statement is a trivial consequence of the first.

We will use the following elementary result to bound the number of trivial composition factors in a module.

Lemma 3.19. Let $G$ be a finite group and $F$ a field of characteristic $p$. Let $M$ be an $F G$-module and let $J$ be a subgroup of $G$.

(1) If $M^{G}=0$ and $G$ can be generated by 2 conjugates of $J$, then $\operatorname{dim} M^{J} \leq$ $(1 / 2) \operatorname{dim} M$.

(2) If $|J|$ is a not a multiple of $p$, then the number of trivial $F G$ composition factors is at most $\operatorname{dim} M^{J}$.

Proof. If $G=\langle J, K\rangle$ for some conjugate $K$ of $J$, then $M^{J} \cap M^{K}=M^{G}=0$, whence (1) holds. In (2), since $J$ has order coprime to the characteristic of $F$, $M=M^{J} \oplus V$ where $J$ has no trivial composition factors on $V$. Thus, the number of $J$-trivial composition factors is at most $\operatorname{dim} M^{J}$ and so this is also an upper bound for the number of $G$-trivial composition factors.

\section{Covering groups}

We will also switch between the simple group and a covering group. Recall that a group $G$ is quasisimple if it is perfect and $G / Z(G)$ is a nonabelian simple group. Recall also the definition of $h^{\prime}(G)$ from (1.3). 
If $N$ is a normal of $G$ and $M$ is a $G$-module with $M^{N}=M$, then we may and do view $M$ as a $G / N$-module.

Lemma 4.1. Let $G$ be a finite quasisimple group. Let $r$ be prime and let $Z$ be a central $r$-subgroup of $G$. Let $M$ be a nontrivial irreducible $F G$-module with $F$ a field of characteristic $p$.

(1) If $M^{Z}=M$, then $H^{1}(G / Z, M) \cong H^{1}(G, M)$.

(2) If $r \neq p$, then either $Z$ acts nontrivially and $H^{2}(G, M)=0$, or $Z$ acts trivially and $H^{2}(G / Z, M) \cong H^{2}(G, M)$.

(3) If $r=p$, then $Z$ acts trivially on $M$, and

$$
\begin{aligned}
\operatorname{dim} H^{2}(G / Z, M) & \leq \operatorname{dim} H^{2}(G, M) \\
& \leq \operatorname{dim} H^{2}(G / Z, M)+c \operatorname{dim} H^{1}(G / Z, M),
\end{aligned}
$$

where $c$ is the rank of $Z$. In particular,

$$
\operatorname{dim} H^{2}(G, M) \leq \operatorname{dim} H^{2}(G / Z, M)+\operatorname{dim} M
$$

(4) $h^{\prime}(G / Z) \leq h^{\prime}(G) \leq h^{\prime}(G / Z)+1$.

Proof. The first statement follows by Lemma 3.8. (2) is included in Lemma 3.12.

So assume that $r=p$.

We use the inequality from Lemma 3.8 (2):

$\operatorname{dim} H^{2}(G, M) \leq H^{2}\left(G / Z, M^{Z}\right)+\operatorname{dim} H^{2}(Z, M)^{G}+\operatorname{dim} H^{1}\left(G / Z, H^{1}(Z, M)\right)$.


Lemma 3.16 since $M^{G}=0$. So the middle term of the right hand side above is 0 . Now $H^{1}(Z, M) \cong \operatorname{Hom}(Z / p Z, M)$. Since $Z / p Z$ is a direct sum of $c$ copies of the trivial $\mathbb{F}_{p} G$-module, where $c$ is at most the rank of $Z, \operatorname{Hom}(Z / p Z, M)$ is isomorphic to $c$ copies of $M$ (as a $G$-module). Thus, $\operatorname{dim} H^{1}\left(G / Z, H^{1}(Z, M)\right) \leq$ $c \operatorname{dim} H^{1}(G / Z, M)$ and so the second inequality in (3) holds. Since $c \leq 2[16$, pp. 313-314], and $\operatorname{dim} H^{1}(G / Z, M) \leq(\operatorname{dim} M) / 2$, the last part of (3) follows.

Finally we show that $\operatorname{dim} H^{2}(G / Z, M) \leq \operatorname{dim} H^{2}(G, M)$. We use relation modules for this purpose. Write $G=F / R$ where $F$ is free of rank $d(G)$. Let $S / R$ be the central subgroup of $F / R$ corresponding to $Z$. Let $R(p)=R /[R, R] R^{p}$ be the $p$-relation module for $G$ and $S(p)=S /[S, S] S^{p}$ the $p$-relation module for $G / Z$. Clearly, there is a $G$-map $\gamma: R(p) \rightarrow S(p)$ with $S(p) / \gamma(R(p))$ having trivial $G$-action. Thus, the multiplicity of an irreducible nontrivial $G$-module $M$ in $S(p) / \operatorname{Rad}(S(p))$ is at most the multiplicity of $M$ in $R(p) / \operatorname{Rad}(R(p))$. Since these multiplicities are $\operatorname{dim} H^{2}(G, M)-\operatorname{dim} H^{1}(G, M)$ and $\operatorname{dim} H^{2}(G / Z, M)-$ $\operatorname{dim} H^{1}(G / Z, M)$, and since, by (1), $\operatorname{dim} H^{1}(G / Z, M)=\operatorname{dim} H^{1}(G, M)$, the inequality follows.

Now (4) follows from (1), (2), (3) and (1.2). 
We can interpret this for profinite presentations. Recall that $\hat{r}(G)$ is the minimal number of relations required among all profinite presentations of the finite group $G$.

Corollary 4.2. Let $G$ be a quasisimple group with a central subgroup $Z$.

(1) $\hat{r}(G / Z) \leq \max \{\hat{r}(G), 2+\operatorname{rank}(J)\} \leq \max \{\hat{r}(G), 4\}$, where $J=H^{2}\left(G / Z, \mathbb{C}^{*}\right)$ is the Schur multiplier of $G / Z$.

(2) $\hat{r}(G) \leq \hat{r}(G / Z)+1$.

Proof. We first prove (1). Let $M$ be an irreducible $G / Z$-module. We may view $M$ as a $G$-module. First suppose that $M$ is trivial. Then $\operatorname{dim} H^{2}(G / Z, M) \leq \operatorname{rank}(J)$.

Now assume that $M$ is nontrivial. Then by Lemma 4.1 (3),

$\operatorname{dim} H^{2}(G, M)-\operatorname{dim} H^{1}(G, M) \geq \operatorname{dim} H^{2}(G / Z, M)-\operatorname{dim} H^{1}(G / Z, M)$.

It follows by (1.1) that either $\hat{r}(G / Z)=2+\operatorname{rank}(J) \leq 4$ or $\hat{r}(G / Z) \leq \hat{r}(G)$, whence the result holds.

We now prove (2). Note that $d(G)=d(G / Z)$. Let $M$ be an irreducible $\mathbb{F}_{p} G$-module which achieves the maximum $\hat{r}(G)$ in (1.1). If $M$ is trivial, then $\operatorname{dim} H^{2}(G / Z, M) \geq \operatorname{dim} H^{2}(G, M)$ and so $\hat{r}(G) \leq \hat{r}(G / Z)$ in this case.

Suppose that $M$ is nontrivial. If $Z$ acts nontrivially on $M$, then $H^{j}(G, M)=0$ for all $j$, a contradiction. So we may assume that $Z$ is trivial on $M$. Then by Lemma 4.1 (4),

$$
\frac{\operatorname{dim} H^{2}(G, M)}{\operatorname{dim} M} \leq \frac{\operatorname{dim} H^{2}(G, M)}{\operatorname{dim} M}+1,
$$

As noted in the previous proof, $\operatorname{dim} H^{1}(G, M)=\operatorname{dim} H^{1}(G / Z, M)$. Now apply (1.1).

The previous two results allow us to work with covering groups rather than simple groups. So if we prove that the universal central extension $G$ of a simple group $S$ can be presented profinitely with $r \geq 4$ relations, the same is true for any quotient of $G$ (and in particular for $S$ ). Conversely, if a finite simple group $S$ can be presented with $r$ profinite relations, then any quasisimple group with central quotient $S$ can be presented with $r+1$ profinite relations.

\section{Faithful irreducible modules and Theorem $\mathrm{C}$}

In this section we show that a bound for $\operatorname{dim} H^{2}(G, M) / \operatorname{dim} M$ with $G$ simple and $M$ a nontrivial irreducible $\mathbb{F}_{p} G$-module implies a related bound for arbitrary finite groups and irreducible faithful modules. In particular, this shows how Theorem B implies Theorem C. 
It is much easier to prove that $\operatorname{dim} H^{1}(G, M) \leq \operatorname{dim} H^{1}(L, M)$ if $M$ is an irreducible faithful $F G$-module and $L$ is any component of $G$. See [18] and Lemma 5.2 (5) below for a stronger result.

For $H^{2}$, the reduction to simple groups is more involved, and it is not clear that the constant one obtains for simple groups is the same constant for irreducible faithful modules. Holt [30] used a similar reduction for a weaker result, but it is not sufficient to appeal to his results.

If $L$ is a nonabelian simple group, let

$$
h_{i}(L)=\max \left\{\operatorname{dim} H^{i}(L, M) / \operatorname{dim} M\right\},
$$

where the maximum is taken over all nontrivial irreducible $\mathbb{F}_{p} L$-modules and all $p$. So $h_{2}(L)=h^{\prime}(L)$ as defined in (1.3). Let $o_{p}(L)$ denote the maximal dimension of any section of $\operatorname{Out}(L)$ that is an elementary abelian $p$-group (this is called the sectional $p$-rank of $\operatorname{Out}(L))$. Let $o(L)=\max _{p}\left\{o_{p}(L)\right\}$. We record some well-known facts about this. See [16, Chapter 4].

Lemma 5.1. Let $L$ be a nonabelian finite simple group. Then $o_{p}(L) \leq 2$ for $p$ odd, and $o(L) \leq 3$.

(1) If $L=A_{n}, n \neq 6$ or $L$ is sporadic, then $\operatorname{Out}(L)$ has order at most 2, and $o(L) \leq 1$.

(2) $\operatorname{Out}\left(A_{6}\right)$ is elementary abelian of order 4.

(3) Assume that $L$ is of Lie type. Then $o_{2}(L) \leq 2$ unless $L \cong \operatorname{PSL}(d, q)$ with $q$ odd and $d>2$ even, or $L \cong P \Omega^{+}(4 m, q)$ with $q$ odd.

Lemma 5.2. Let $F$ be a field of characteristic $p, G$ a finite group and $M$ an irreducible $F G$-module that is faithful for $G$. Assume that $H^{k}(G, M) \neq 0$ for some $k>0$ (and so in particular, $p>0)$.

(1) $O_{p}(G)=O_{p^{\prime}}(G)=1$; in particular $G$ is not solvable.

(2) Let $N=\mathrm{F}^{*}(G)$. For some $t \geq 1, N$ is a direct product of t nonabelian simple groups.

(3) G has at most $k$ minimal normal subgroups.

(4) If $W$ is an irreducible $F N$-submodule of $M$, and if two distinct components of $G$ act nontrivially on $W$, then $H^{1}(G, M)=0$ and $\operatorname{dim} H^{2}(G, M) \leq(\operatorname{dim} M) / 4$. In particular, this is the case if $G$ does not have a unique minimal normal subgroup.

(5) Suppose that $N$ is the unique minimal normal subgroup of $G$ and $L$ is a component of $G$.

(a) $\operatorname{dim} H^{1}(G, M) \leq \operatorname{dim} H^{1}(L, W)$ for $W$ any irreducible $L$-submodule of $M$ with $W^{L}=0$. 
(b) $\operatorname{dim} H^{2}(G, M) \leq\left(h_{1}(L)(o(L)+1 / 2)+h_{2}(L) / t\right) \operatorname{dim} M$.

(c) If $N=L$, then $\operatorname{dim} H^{2}(G, M) \leq\left(h_{2}(L)+1\right) \operatorname{dim} M$.

Proof. The hypotheses imply that $M$ is not projective and so $p>0$. Since $M$ is faithful and irreducible, $O_{p}(G)=1$. If $O_{p^{\prime}}(G) \neq 1$, then by Corollary 3.12, $H^{d}(G, M)=0$ for all $d$. So (1) and (2) hold.

We may assume that $F$ is algebraically closed (see Lemma 3.2). Write $N=$ $N_{1} \times \cdots \times N_{e}$ where the $N_{i}$ are the minimal normal subgroups of $G$. Let $W$ be an irreducible $F N$-submodule. Then $W=W_{1} \otimes \cdots \otimes W_{e}$ is a tensor product of irreducible $F N_{i}$-modules. Since $M$ is faithful and irreducible, $M^{N_{i}}=0$. In particular, each $N_{i}$ is faithful on $W$, whence $W_{i}$ is nontrivial for each $i$.

It follows by Lemma 3.10, that $H^{j}(N, W)=0$ for $j<e$. So $H^{j}(N, g W)=0$ for any $g \in G$ with $j<e$. Since $M$ is a direct sum of irreducible $N$-modules of the form $g W, g \in G, H^{j}(N, M)=0$ for $j<e$. It follows by Lemma 3.11 that $H^{j}(G, M)$ embeds in $H^{j}(N, M)=0$ for $j<e$, whence (3) follows (see also [49]).

The same argument shows that $H^{j}(G, M)=0$ if there is an irreducible $F N$ submodule $W$ of $G$ in which at least $j+1$ components act nontrivially. If precisely $j$ components act nontrivially, the argument shows that $\operatorname{dim} H^{j}(G, M) \leq$ $(\operatorname{dim} M) / 2^{j}$. Since $N_{i}$ has no fixed points on $M$, it follows that at least $e$ components act nontrivially on any irreducible $F N$-submodule, whence (4) holds.

So assume that $N$ is the unique minimal normal subgroup of $G$. Write $N=$ $L_{1} \times \cdots \times L_{t}$ with the $L_{i}$ isomorphic nonabelian simple groups. Set $L=L_{1}$ and $h_{1}=h_{1}(L)$.

Let $W$ be an irreducible $F N$-submodule with $W^{L}=0$. Suppose first that $L_{j}$ acts nontrivially on $W$ for some $j>1$. Arguing as above and using Lemma 3.10 and Lemma 3.8 shows that $H^{1}(G, M)=H^{1}(N, M)=0$. Similarly, we see that $\operatorname{dim} H^{2}(N, W) \leq h_{1}^{2} \operatorname{dim} W$ and $\operatorname{dim} H^{2}(G, M) \leq \operatorname{dim} H^{2}(N, M) \leq h_{1}^{2} \operatorname{dim} M$. Using (1.2) shows that (5) (b) follows in this case.

So to complete the proof of all parts of (5), we may assume that $L_{j}$ is trivial on $W$ for $j>1$ (for case (5) (c), there is only one component).

We now prove the first part of (5). Let $U$ be the largest $L$-homogeneous submodule of $M$ containing $W$ (i.e. $U$ is the $L$-submodule generated by the $L$-submodules isomorphic to $W$ ).

Let $I$ be the stabilizer of $U$ in $G$. Note that $I \leq N_{G}(L)$ (since for $j \neq 1$, $L_{j}$ acts trivially on $U$ ). Since $M$ is irreducible, $U$ is an irreducible $I$-module. Let $R=L C_{I}(L)$. By Lemma 3.4, $H^{k}(G, M) \cong H^{k}(I, U)$. By Lemma 3.11, $\operatorname{dim} H^{1}(I, U) \leq \operatorname{dim} H^{1}(R, U)$. Since $R=L \times C_{I}(L), U$ is a direct sum of modules of the form $W \otimes X$ where each $X$ is an irreducible $C_{I}(L)$-module. Since $W$ is irreducible, it follows that either all $X$ are trivial $C_{I}(L)$-modules or none are. In the latter case, $H^{1}(R, W)=0$ by Lemma 3.10 , and so $H^{1}(G, M)=0$. So $C_{I}(L)$ acts trivially on $U$. Set $J=I / C_{I}(L)$. 
By Lemma 3.8, $\operatorname{dim} H^{1}(I, U) \leq \operatorname{dim} H^{1}(J, U)+\operatorname{dim} H^{1}\left(C_{I}(L), U\right)^{I}$. Since $C_{I}(L)$ is trivial on $W, H^{1}\left(C_{I}(L), W\right)^{I}$ is the set of $I$ - homomorphisms from $C_{I}(L)$ to $U$. Since $L$ acts trivially on $C_{I}(L)$ and $U^{L}=0, H^{1}\left(C_{I}(L), U\right)^{I}=0$. Thus $\operatorname{dim} H^{2}(I, U) \leq \operatorname{dim} H^{2}(J, U)$. Note that $J$ is almost simple with socle $L$ and that $J$ acts irreducibly on $U$. So we have reduced the problem to the case $t=1$, $C_{G}(L)=1$ and $G=I$. Now use the fact that $G / L$ is solvable (which depends on the classification of finite simple groups) and let $D / L$ be a maximal normal subgroup of $I / L$. So $I / D$ is cyclic of prime order $s$. If $D$ does not act homogeneously, then $U$ is induced and we can apply Lemma 3.4. So we may assume that $D$ acts homogeneously. It follows by Clifford theory and the fact that $I / D$ is cyclic that $D$ acts irreducibly on $U$. By Lemma 3.11, $\operatorname{dim} H^{1}(I, U) \leq \operatorname{dim} H^{1}(D, U)^{I} \leq \operatorname{dim} H^{1}(D, U)$, and so by induction (on $|I: L|), \operatorname{dim} H^{1}(D, U) \leq \operatorname{dim} H^{1}(L, W)$, as required.

Now consider $H^{2}(G, M)$ in (5). Let $M_{i}=\left[L_{i}, M\right]$. So $M$ is the direct sum of the $M_{i}$. Another application of Lemma 3.8, together with the fact that $M^{N}=0$, shows that

$$
\operatorname{dim} H^{2}(G, M) \leq \operatorname{dim} H^{2}(N, M)^{G}+\operatorname{dim} H^{1}\left(G / N, H^{1}(N, M)\right) .
$$

Now $H^{2}(N, M)$ is the direct sum of the $H^{2}\left(N, M_{i}\right) \cong H^{2}\left(L_{i}, M_{i}\right)$ (by Lemma 3.10), and $G$ permutes these terms transitively. Thus $\operatorname{dim} H^{2}(N, M)^{G} \leq \operatorname{dim} H^{2}\left(L, M_{1}\right) \leq$ $\left(h_{2}(L) / t\right) \operatorname{dim} M$.

Similarly, $H^{1}(N, M)$ is the direct sum of the $H^{1}\left(L_{i}, M_{i}\right)$, and $G / N$ permutes these. Thus, $H^{1}(N, M)$ is an induced $G / N$-module, and so by Shapiro's Lemma (Lemma 3.4) $H^{1}\left(G / N, H^{1}(N, M)\right) \cong H^{1}\left(N_{G}(L) / N, H^{1}\left(L, M_{1}\right)\right)$. Note that $M_{1}$ is an irreducible $N_{G}(L)$-module and is a faithful $L$-module (since $M$ is irreducible for $G$ ).

Let $P$ be a Sylow $p$-subgroup of $N_{G}(L)$. Let $K=\cap_{i} N_{P}\left(L_{i}\right)$ and note that $K$ is normal in $P$. Then $K N / N$ can be generated by $o(L) t$ elements (by induction on $t$ ). By [4, Theorem 2.3], $P / K$ can be generated by $\lfloor t / 2\rfloor$ elements, whence $P N / N$ can be generated by at most $(o(L)+1 / 2) t$ elements. Since $\operatorname{dim} H^{1}\left(L, M_{1}\right) \leq$ $h_{1}(L)\left(\operatorname{dim} M_{1}\right)$, it follows that

$$
\begin{aligned}
\operatorname{dim} H^{1}\left(N_{G}(L) / N, H^{1}\left(L, M_{1}\right)\right) & \leq \operatorname{dim} H^{1}\left(P N / N, H^{1}\left(L, M_{1}\right)\right) \\
& \leq h_{1}(L)(o(L)+1 / 2) t \operatorname{dim} M_{1} \\
& =h_{1}(L)(o(L)+1 / 2) \operatorname{dim} M .
\end{aligned}
$$

Thus,

This gives (5) (b).

$$
\frac{\operatorname{dim} H^{2}(G, M)}{\operatorname{dim} M} \leq h_{1}(L)(o(L)+1 / 2)+h_{2}(L) / t
$$

We now prove (5) (c). So assume that $t=1$. Then $P N / N$ can be generated by $o(L)$ elements and so we get the bound $\operatorname{dim} H^{2}(G, M) / \operatorname{dim} M \leq h_{2}(L)+$ $o(L) h_{1}(L)$. 
By (1.2), $h_{2}(L)+o(L) h_{1}(L) \leq h_{2}(L)+1$ unless $o(L)>2$. However, we have already noted that in the cases where $o(L)=3, L$ must be a group of Lie type over a field of odd characteristic and the $p$-subgroup of $\operatorname{Out}(L)$ requiring 3 generators must be a 2-subgroup. It follows by [28] that in all these cases $\operatorname{dim} H^{1}(L, M) \leq$ $(\operatorname{dim} M) / 3$, whence the result holds.

Theorem 5.3. Let $F$ be a field, $G$ be a finite group with $M$ a faithful irreducible $F G$-module. If $H^{2}(G, M) \neq 0$, then $G$ has a component $L$ and

$$
\frac{\operatorname{dim} H^{2}(G, M)}{\operatorname{dim} M} \leq \max \left\{7 / 4, h_{2}(L)+1\right\} .
$$

Proof. Since $H^{2}(G, M) \neq 0$, the previous lemma applies. If $G$ has more than one minimal normal subgroup, then $\operatorname{dim} H^{2}(G, M) \leq(\operatorname{dim} M) / 4$ by Lemma $5.2(4)$, and the result holds. So we may assume that $G$ has a unique minimal normal subgroup $N$, that $L$ is a component of $G$ and that $N$ is a direct product of $t$ conjugates of $L$. Now the bound in (5) (b) of the previous lemma applies.

As we have noted above, $o(L) \leq 3$ with equality implying that $G$ is a finite group of Lie type $A$ of rank at least 3 or of type $D$ of rank at least 4. If $o(L) \leq 2$, it follows from (1.2) that $h_{1}(L)(o(L)+1 / 2) \leq 7 / 4$. If $o(L)=3$, it follows from [19] that $h_{1}(L) \leq 1 / 3$, whence $h_{1}(L)(o(L)+1 / 2) \leq 5 / 4$.

Let $t$ be the number of components of $G$. If $t=1$, the result follows by (5)(c) of the previous lemma. So assume that $t>1$. By (5)(b) of the previous result, $\operatorname{dim} H^{2}(G, M) / \operatorname{dim} M \leq h_{1}(L)(o(L)+1 / 2)+h_{2}(L) / 2$. The right hand side is bounded above by $(5 / 4)+h_{2}(L) / 2 \leq \max \left\{7 / 4, h_{2}(L)+1\right\}$, and the result follows.

An immediate consequence of the previous result is:

Corollary 5.4. Theorem B implies Theorem $C$.

\section{Alternating and symmetric groups}

We will need the following better bound for $H^{1}$ for alternating groups given in [23].

Theorem 6.1. Let $G=A_{n}, n>4$. If $F$ is a field and $M$ is an irreducible $F G$ module, then

(1) $\operatorname{dim} H^{1}(G, M) \leq(\operatorname{dim} M) /(f-1)$ where $f$ is the largest prime such that $f \leq n-2$;

(2) $\operatorname{dim} H^{1}(G, M) \leq(2 / n) \operatorname{dim} M$ for $n>8$;

(3) $\operatorname{dim} H^{1}\left(A_{8}, M\right) \leq(\operatorname{dim} M) / 6$; and 
(4) if $F$ has characteristic $p$, then $\operatorname{dim} H^{1}(G, M) \leq(\operatorname{dim} M) /(p-2)$.

The goal of this section is to prove the following results:

Theorem 6.2. Let $G=A_{n}$ or $S_{n}, n>4$, and let $p$ be a prime. Let $F$ be a field of characteristic $p$ and $M$ an $F G$-module.

(1) If $p>3$, then $\operatorname{dim} H^{2}(G, M) \leq(\operatorname{dim} M) /(p-2) \leq(\operatorname{dim} M) / 3$.

(2) If $p=3$, then $\operatorname{dim} H^{2}(G, M) \leq \operatorname{dim} M$ with equality if and only if $n=6$ or 7 and $M$ is the trivial module.

(3) If $p=2$, then $\operatorname{dim} H^{2}\left(A_{n}, M\right) \leq(35 / 12) \operatorname{dim} M$.

(4) If $p=2$, then $\operatorname{dim} H^{2}\left(S_{n}, M\right)<3 \operatorname{dim} M$.

These results are likely quite far from best possible. By (1.1) and Corollary 4.2, this gives:

Corollary 6.3. (1) $\hat{r}\left(A_{n}\right) \leq 4$,

(2) $\hat{r}\left(S_{n}\right) \leq 4$, and

(3) if $G$ is any quasisimple group with $G / Z(G)=A_{n}$, then $\hat{r}(G) \leq 5$.

Almost certainly, it is the case that $\hat{r}\left(A_{n}\right)=\hat{r}\left(S_{n}\right)=3$ for $n>4$. Since the Schur multipliers of $A_{n}$ and $S_{n}$ are nontrivial for $n>4, \hat{r}\left(A_{n}\right)$ and $\hat{r}\left(S_{n}\right)$ are both at least 3 . The proof we give says very little about finding specific relations. It would be quite interesting to pursue this further.

The main idea is to pass to a subgroup containing a Sylow $p$-subgroup of $G$ and having a normal subgroup that is a direct product of alternating groups. We then use induction together with the results of Section 3.

We do this first for $p>3$, then for $p=3$ and finally for $p=2$. If $p>3$, each of these smaller alternating groups is simple and has Schur multiplier prime to $p$. If $p=3$, there may be an $A_{3}$ factor. Also, $A_{6}$ and $A_{7}$ have Schur multipliers of order 6 . For $p=2$, there may be solvable factors, all Schur multipliers have even order and there are further complications as well.

6.1. $p>3$. For this subsection, let $F$ be an algebraically closed field of characteristic $p>3$. Our goal is to prove the following result, which includes Theorem 6.2 for $p>3$.

Theorem 6.4. Let $p>3$ be a prime. Let $G=A_{n}$ and $F$ a field of characteristic $p$. If $M$ is an $F G$-module, then $\operatorname{dim} H^{j}(G, M) \leq(\operatorname{dim} M) /(p-2)$ for $j=1,2$. 
Proof. We induct on $n$. If $p<n$, all $F G$-modules are projective and so $H^{j}(G, M)=0$ for $j>0$.

If $p$ does not divide $n$, then the restriction map from $H^{j}\left(A_{n}, M\right)$ to $H^{j}\left(A_{n-1}, M\right)$ is injective by Lemma 3.6 and the result follows. So we may assume that $p \mid n$. Since $G$ is perfect, Lemma 3.9 implies that $H^{1}(G, F)=0$, and since $p$ does not divide the order of the Schur multiplier of $G, H^{2}(G, F)=0$.

As usual, we may assume that $M$ is an irreducible $F G$-module.

If $n=p$, then by Lemma 3.5, $\operatorname{dim} H^{j}(G, M) \leq 1$. Thus, the result follows by noting that $\operatorname{dim} M \geq p-2$ for every nontrivial irreducible $G$-module.

Suppose that $n \neq p^{a}$ for any $a$. Write $n=p^{a}+n^{\prime}$ where $p^{a}$ is the largest power of $p$ less than $n$. Set $H=A_{p} a \times A_{n^{\prime}}<G$. Since $H$ contains a Sylow $p$-subgroup of $G$, it suffices to show by Lemma 3.6 that $H$ satisfies the conclusion of the theorem. So let $V=V_{1} \otimes V_{2}$ be an irreducible $F H$-module. If $V$ is a trivial $H$-module, then $H^{j}(H, V)=0$ for $j=1,2$ (by Lemma 3.9). Otherwise, the result follows by Lemma 3.10 and induction.

Finally suppose that $n=p^{a+1}>p$. Let $H=A_{p^{a}} \prec A_{p}<G$. Then $H$ contains a Sylow $p$-subgroup of $G$ and again it suffices to show that the conclusion holds for $H$. Let $V$ be an irreducible $F H$-module. Let $N$ be the normal subgroup of $H$ with $H / N \cong A_{p}$. So $N=L_{1} \times \cdots \times L_{p}$ where $L_{i} \cong A_{p^{a}}$. The result is straightforward and easier for $H^{1}$, and we just give the argument for $H^{2}$. By Lemma 3.8,

$$
\begin{gathered}
\operatorname{dim} H^{2}(H, V) \leq \operatorname{dim} H^{2}\left(H / N, V^{N}\right)+\operatorname{dim} H^{2}(N, V)^{H} \\
+\operatorname{dim} H^{1}\left(H / N, H^{1}(N, V)\right) .
\end{gathered}
$$

If $N$ is trivial on $V$, then the last two terms are 0 and the result holds since we already know the theorem for $n=p$. So suppose that $V^{N}=0$. Let $W$ be an irreducible $F N$-submodule of $V$. So $W=W_{1} \otimes \cdots \otimes W_{p}$, where $W_{i}$ is an irreducible $F L_{i}{ }^{-}$ module. By Lemma 3.10, $H^{1}(N, W)=0$ unless (after reordering if necessary) $W_{1}$ is nontrivial and $W_{j}$ is trivial for each $j>1$. Suppose that for some $j>1, W_{j}$, is nontrivial. Thus, by Lemma 3.10, $H^{1}(N, W) \cong H^{1}(N, g W)=0$ for every $g \in G$ Since $W$ is a direct sum of $N$-submodules of the form $g W, g \in G$, this implies that $H^{1}(N, V)=0$. By Lemma 3.11 and induction, $\operatorname{dim} H^{2}(H, V) \leq$ $\operatorname{dim} H^{2}(N, V)^{H} \leq(\operatorname{dim} V) /(p-2)$.

Now suppose that $W_{1}$ is nontrivial and $W_{j}$ is trivial for all $j>1$. Let $W_{1} \leq$ $M_{1}$ be the set of fixed points of $L_{2} \times \cdots \times L_{p}$ on $V$. The stabilizer of $M_{1}$ is clearly $N_{H}\left(L_{1}\right)$, and so $V$ is induced from $M_{1}$. By Shapiro's Lemma (Lemma 3.4), $H^{2}(H, M) \cong H^{2}\left(N_{H}\left(L_{1}\right), M_{1}\right)$. Since $\left[N_{H}\left(L_{1}\right): N\right]$ is prime to $p$, it follows by Lemma 3.12 that $\operatorname{dim} H^{2}(H, M) \leq \operatorname{dim} H^{2}\left(N, M_{1}\right)$. By Lemma 3.10 and the fact that $H^{1}\left(L_{j}, M_{1}\right)=0$ for $j>0, H^{2}\left(N, M_{1}\right)=H^{2}\left(L_{1}, M_{1}\right)$ and the result follows. 
6.2. $p=3$

Theorem 6.5. Let $G=A_{n}, n>2$ and $F$ a field of characteristic $p=3$. Let $M$ be an irreducible $F G$-module.

(1) If $M$ is trivial, then $H^{2}(G, M)=0$ unless $n=3,4,6$ or 7 , in which case $H^{2}(G, M)$ is 1-dimensional.

(2) If $n=3^{a}>3$, then $\operatorname{dim} H^{2}(G, M) \leq(3 / 5) \operatorname{dim} M$.

(3) If $M$ is nontrivial, then $\operatorname{dim} H^{2}(G, M) \leq(21 / 25) \operatorname{dim} M$.

Proof. The proof proceeds as in the previous result. However, note that if $M$ is the trivial module, then $H^{2}(G, M)$ is 1-dimensional for $n=3,4,6$ and 7 and 0 otherwise (for $n \geq 5$, see [16, p. 314] and for $n=3$ or 4, a Sylow 3-subgroup is cyclic). We use Lemma 3.6 extensively and sometimes without comment.

So assume that $M$ is irreducible and nontrivial. There is no loss in assuming that $F$ is algebraically closed.

If $n \leq 12$, the result is in [42]. By induction, using Lemma 3.6, we may assume that $n$ is divisible by 3 (since $n>12$ and (1) implies that trivial modules are not an exception to the bound).

Case 1. $n=3^{a+1}>9$.

Let $N:=H_{1} \times H_{2} \times H_{3}$ where each $H_{i}=A_{3} a$. Let $H$ be a subgroup of the normalizer of $N$ with $H / N \cong A_{3}$. Note that $H$ contains a Sylow 3-subgroup of $G$ and so Lemma 3.6 applies.

Let $V$ be an absolutely irreducible $F H$-module. If $V^{N}=V$, then by Lemma 3.8,

$$
\begin{gathered}
\operatorname{dim} H^{2}(H, V) \leq \operatorname{dim} H^{2}(H / N, V)+\operatorname{dim} H^{2}(N, V)^{H} \\
+\operatorname{dim} H^{1}\left(H / N, H^{1}(N, V)\right) .
\end{gathered}
$$

Since $3^{a}>3$, the Schur multiplier of $N$ is a 2-group and so the middle term above is 0 . Since $N$ is perfect, the last term above is 0 , and so $\operatorname{dim} H^{2}(H, V) \leq 1$ by Lemma 3.5.

Suppose that $V^{N}=0$. Let $W$ be an irreducible $F N$-submodule of $V$. Write $W=W_{1} \otimes W_{2} \otimes W_{3}$ where $W_{i}$ is an $F H_{i}$ irreducible module. We may assume that $W_{1}$ is nontrivial. By Lemma 5.3, either $\operatorname{dim} H^{2}(H, V) \leq \operatorname{dim} H^{2}(N, V) \leq \operatorname{dim} V / 4$ or $W_{2}$ and $W_{3}$ are trivial. Thus, $V=X_{N}^{H}$ where $X$ is the fixed space of $H_{2} \times H_{3}$. By Lemma 3.4, it follows that $H^{2}(H, V) \cong H^{2}(N, X)$. By Lemma 3.10, $H^{2}(N, X) \cong$ $H^{2}\left(H_{1}, X\right)$. By induction, $\operatorname{dim} H^{2}(H, V) \leq(3 / 5) \operatorname{dim} X=(1 / 5) \operatorname{dim} V$.

We claim that the number $d$ of trivial composition factors of $N$ on $M$ is at most $(\operatorname{dim} M) / 2$ (in fact, it is usually much less). Let $T$ be a Sylow 2-subgroup of $N$. It is easy to see that some pair of conjugates of $T$ generate $G$. So we see by Lemma 3.19 that $d \leq \operatorname{dim} M^{T} \leq(\operatorname{dim} M) / 2$. The previous paragraphs show that $\operatorname{dim} H^{2}(H, M) \leq$ $d+(\operatorname{dim} M-d) / 5$. Since $d \leq(\operatorname{dim} M) / 2$, this implies that $\operatorname{dim} H^{2}(H, M) \leq$ $(\operatorname{dim} M) / 2+(\operatorname{dim} M) / 10=(3 / 5) \operatorname{dim} M$ as required. 
Case 2. $n$ is not of the form $3^{a}+3$ or $3^{a}+6$.

We may assume also that $n \neq 3^{a}$ (by case 1). So $n=n_{1}+n_{2}$ where $n_{1}=3^{a}>$ $n / 3$ and $n_{2} \geq 9$. Set $H=H_{1} \times H_{2}<G$ where the $H_{i}$ are alternating groups of degree $n_{i}$. Note that $[G: H]$ has index prime to 3. By induction and Lemma 3.10, the result follows.

Case 3. $n=3^{a}+3>12$.

Let $H=H_{1} \times H_{2}<G$ with $H_{1}=A_{3}$ and $H_{2}=A_{3} a$. Let $V$ be an irreducible $F H$-module. Then $V$ is trivial for $H_{1}$ (since it is a normal 3-subgroup of $H)$ and irreducible for $H_{2}$. If $V$ is nontrivial then, by Lemma $3.10, H^{2}(H, V) \cong$ $H^{1}\left(H_{2}, V\right) \oplus H^{2}\left(H_{2}, V\right)$. By Theorem 6.1 and the fact that $n_{1} \geq 27$, the first term has dimension at most $(1 / 23) \operatorname{dim} V$ and the second has dimension at most (3/5) $\operatorname{dim} V$ by induction. Thus, $\operatorname{dim} H^{2}(H, V)<(17 / 25) \operatorname{dim} M$.

If $V$ is trivial, then $\operatorname{dim} H^{2}(H, V)=1$. Arguing as above, we see that the number of $H$-trivial composition factors in $M$ is at most $(\operatorname{dim} M) / 2$ and so

$$
\frac{\operatorname{dim} H^{2}(G, M)}{\operatorname{dim} M} \leq \frac{1+17 / 25}{2}=\frac{21}{25} .
$$

Case 4. $n=3^{a}+6 \geq 15$.

The proof is quite similar to the previous case.

Let $H=H_{1} \times H_{2}$ where $H_{1} \cong A_{3} a$ and $H_{2} \cong A_{6}$. Then $H$ contains a Sylow 3 -subgroup of $G$. So it suffices to prove the bound for $H$. Let $V$ be an irreducible $F H$-module. So $V=V_{1} \otimes V_{2}$ where $V_{i}$ is an irreducible $F H_{i}$-module for $i=1,2$. If both $V_{i}$ are nontrivial, then $\operatorname{dim} H^{2}(H, V)=\operatorname{dim} H^{1}\left(H_{1}, V_{1}\right) \cdot \operatorname{dim} H^{1}\left(H_{2}, V_{2}\right)$. The first term is at most $(1 / 7) \operatorname{dim} V_{1}$ by Theorem 6.1 and the second is at most $(1 / 2) \operatorname{dim} V_{2}$, and so $\operatorname{dim} H^{2}(H, V) \leq(1 / 14) \operatorname{dim} M$. If $V_{1}$ nontrivial and $V_{2}$ is trivial, then by Lemma $3.10, H^{2}(H, V) \cong H^{2}\left(H_{1}, V_{1}\right) \leq(3 / 5) \operatorname{dim} M$. If $V_{1}$ is trivial, then $\operatorname{dim} H^{2}\left(A_{6}, V_{2}\right) \leq \operatorname{dim} V_{2}$. As in the previous case, the number of trivial composition factors for $H_{1}$ is at most $(\operatorname{dim} M) / 2$, and the result follows as in the previous case.

6.3. $\boldsymbol{p}=2$. Let $F$ be an algebraically closed field of characteristic 2 . In this section, all modules are over $F$. Let $n \geq 5$ be a positive integer. Write $n=\sum_{i=1}^{r} 2^{a_{i}}$, where the $a_{i}=a_{i}(n)$ are decreasing positive integers.

The next result follows since the 2-part of the Schur multiplier for $A_{n}$ has order 2 [16, p. 312].

Lemma 6.6. Let $M$ be the trivial module.

(1) $\operatorname{dim} H^{2}\left(A_{n}, M\right)=1$; and

(2) $\operatorname{dim} H^{2}\left(S_{n}, M\right)=2$.

Lemma 6.7. Let $M$ be an irreducible nontrivial $F S_{n}$-module for $n \geq 8$. Then 
(1) $\operatorname{dim} H^{2}\left(S_{n}, M\right) \leq \operatorname{dim} H^{2}\left(A_{n}, M\right)+\operatorname{dim} M / 2^{a_{1}(n)-1}$.

(2) $\operatorname{dim} H^{2}\left(S_{n}, M\right) \leq \operatorname{dim} H^{2}\left(A_{n}, M\right)+\operatorname{dim} M / 13$ for $n \geq 12$.

Proof. By Lemma 3.8, we have

$$
\operatorname{dim} H^{2}\left(S_{n}, M\right) \leq \operatorname{dim} H^{2}\left(A_{n}, M\right)^{S_{n}}+\operatorname{dim} H^{1}\left(S_{n} / A_{n}, H^{1}\left(A_{n}, M\right)\right) .
$$

By Theorem 6.1, the right hand term is at most $\operatorname{dim} H^{1}\left(A_{n}, M\right) \leq \operatorname{dim} M /(f-1)$ where $f$ is the largest prime with $f \leq n-2$. By Bertrand's postulate, there is a prime $f$ with $a_{1}(n) \leq n / 2 \leq f-1 \leq n-2$, whence (1) holds. Now (2) holds by the same argument for $n \geq 15$ (since 13 is prime and $13 \leq n-2$ ), and by computation for $n \leq 14$ [42].

Lemma 6.8. Let $n=2^{a+1} \geq 4$. Let $G=A_{n}$ or $S_{n}$. Let $F$ be an algebraically closed field of characteristic 2. Let $M$ be an irreducible nontrivial $F G$-module.

(1) If $n=4$ and $G=A_{n}$, then $\operatorname{dim} H^{2}(G, M) \leq 1$.

(2) If $n=4$ and $G=S_{n}$, then $\operatorname{dim} H^{2}(G, M) \leq 1$.

(3) If $n \geq 8$ and $G=A_{n}$, then $\operatorname{dim} H^{2}(G, M) \leq(65 / 24) \operatorname{dim} M$.

(4) If $n \geq 8$ and $G=S_{n}$, then $\operatorname{dim} H^{2}(G, M)<(17 / 6) \operatorname{dim} M$.

Proof. If $n \leq 8$, this is done by a computer computation in [42]. So assume that $n \geq 16$. We induct on $n$. By (2) of the previous lemma, it suffices to prove (3). As usual, we will compute cohomology for subgroups which contain a Sylow 2-subgroup. So we may use Lemma 3.6.

Let $N=A_{2} a \times A_{2^{a}}=H_{1} \times H_{2}$ and $H$ be the normalizer in $G$ of $N$. Let $V$ be an irreducible $F H$-module. If $V$ is trivial, it follows by Lemma 3.8 that $\operatorname{dim} H^{2}(H, V) \leq$ $\operatorname{dim} H^{2}(H / N, V)+\operatorname{dim} H^{2}(N, V)^{H}+\operatorname{dim} H^{1}\left(H / N, H^{1}(N, V)\right)$. Since $N$ is perfect, the last term is 0 . Since $H / N$ has order 4 , the first term on the right side of the inequality is 3. Since the Schur multiplier of each factor of $N$ has order 2, $H^{2}(N, V)$ is 2-dimensional and $H$ acts nontrivially on this, whence the middle term has dimension 1 . Thus, $\operatorname{dim} H^{2}(H, V) \leq 4$.

Suppose that $V$ is nontrivial. Then $V^{N}=0$. Let $W$ be an irreducible $N$ submodule of $V$. Write $W=W_{1} \otimes W_{2}$. Note that $V$ is a direct sum of $N$-modules of the form $g W, g \in H$. If both $W_{1}$ and $W_{2}$ are nontrivial, then by Lemma 3.10, $H^{2}(N, W)=H^{1}\left(H_{1}, W_{1}\right) \otimes H^{1}\left(H_{2}, W_{2}\right)$ and $H^{1}(N, V)=0$. By Theorem 6.1 and Lemma 3.8, $\operatorname{dim} H^{2}(H, V) \leq \operatorname{dim} H^{2}(N, V)^{H} \leq(\operatorname{dim} V) / 36$.

If $W_{2}$ is trivial, then $V$ is an induced module - so we may write $V=U_{J}^{H}$ where $J=N$ or $H / J$ has order 2. Thus, by Lemma $3.4, H^{2}(H, V) \cong H^{2}(J, U)$. If $J=N$, this implies that $\operatorname{dim} H^{2}(J, U)=\operatorname{dim} H^{2}\left(H_{1}, U\right)$. So by induction, 
$\operatorname{dim} H^{2}(J, U) \leq(65 / 24) \operatorname{dim} U$, whence $\operatorname{dim} H^{2}(H, V) \leq(65 / 96) \operatorname{dim} V$. If $J / N$ has order 2, then we apply Lemma 3.8 to conclude that

$$
\begin{gathered}
\operatorname{dim} H^{2}(J, U) \leq \operatorname{dim} H^{2}\left(J / N_{2}, U\right)+\operatorname{dim} H^{2}\left(H_{2}, U\right)^{J} \\
+\operatorname{dim} H^{1}\left(J / H_{2}, H^{1}\left(N_{2}, U\right)\right) .
\end{gathered}
$$

Since $H_{2}$ is perfect and $U$ is trivial for $H_{2}$, the last term is 0 . Since $H_{2}$ is trivial on $U$ and $H^{2}\left(N_{2}, F\right)$ is 1-dimensional, we see that $H^{2}\left(N_{2}, U\right) \cong U$ as a $J$-module and so $J$ has no fixed points on the module, whence the middle term is 0 . Noting that $J / H_{2} \cong S_{2} a$ and using Lemma 6.7 and induction, we conclude that $\operatorname{dim} H^{2}(J, U) \leq$ $(17 / 6) \operatorname{dim} U$. Since $\operatorname{dim} U=(\operatorname{dim} V) / 2$, we obtain the desired inequality that $\operatorname{dim} H^{2}(H, V) \leq(17 / 12) \operatorname{dim} V$.

Note $H$ contains an element $h$ that is the product of two disjoint cycles of length $2^{a}-1$. It is easy to see that $A_{n}$ can be generated by two conjugates of $h$. Setting $J=$ $\langle h\rangle$ and using Lemma 3.19, we see that the number $\alpha$ of trivial $H$-composition factors in $M$ is at most $(\operatorname{dim} M) / 2$. Thus, $\operatorname{dim} H^{2}(H, M) \leq 4 \alpha+(17 / 12)(\operatorname{dim} M-\alpha)$. Since $\alpha \leq(\operatorname{dim} M) / 2$, this implies that $\operatorname{dim} H^{2}(H, M) \leq(65 / 24) \operatorname{dim} M$.

Theorem 6.9. Let $n>4$ be a positive integer and $F$ an algebraically closed field of characteristic 2. Let $M$ be an FG-module with $G=A_{n}$ or $S_{n}$.

(1) $\operatorname{dim} H^{2}\left(A_{n}, M\right) \leq(35 / 12) \operatorname{dim} M$; and

(2) $\operatorname{dim} H^{2}\left(S_{n}, M\right)<3 \operatorname{dim} M$.

Proof. If $M$ is trivial, then $\operatorname{dim} H^{2}\left(A_{n}, M\right)=1$ since a Sylow 2-subgroup of the Schur multiplier has order 2. Since $S_{n} / A_{n}$ has order 2, it follows by Lemma 3.8 that $\operatorname{dim} H^{2}\left(S_{n}, M\right) \leq 2$, whence the result holds in this case. So we may assume that $M$ is a nontrivial irreducible $F G$-module. If $n \leq 14$, then (1) and (2) follow by computation [42]. So we assume that $n>14$.

By Lemma 6.7, it suffices to prove the results for $A_{n}$. So assume that $G=A_{n}$ and $M$ is a nontrivial irreducible $F G$-module.

The result holds for $n$ a power of 2 by the previous lemma. So assume that this is not the case. As usual, we will obtain bounds for a subgroup of odd index and then apply Lemma 3.6. We may also assume that $n$ is even (since if not, $A_{n-1}$ has odd index). Thus, we may write $n=n_{1}+n_{2}$ where $n_{1}=2^{a}$ is the largest power of 2 less than $n$ and $n_{2} \geq 2$.

First suppose that $n_{2}=2$. Then $S_{2} a$ contains a Sylow 2-subgroup of $G$ and so by the previous lemma, $\operatorname{dim} H^{2}(G, M) \leq(17 / 6) \operatorname{dim} M$. So we assume that $n_{2} \geq 4$.

Let $N=N_{1} \times N_{2}$ where $N_{i} \cong A_{n_{i}}$ and let $H$ be the normalizer of $N$. Then $H / N$ has order 2 and $H$ contains a Sylow 2-subgroup of $G$.

Let $V$ be an irreducible $F H$-module. If $V$ is trivial, then by Lemma 3.8,

$$
\begin{gathered}
\operatorname{dim} H^{2}(H, V) \leq \operatorname{dim} H^{2}(H / N, V)+\operatorname{dim} H^{2}(N, V)^{H} \\
+\operatorname{dim} H^{1}\left(H / N, H^{1}(N, V)\right) .
\end{gathered}
$$


The first term on the right hand side is 1 . Since $N=N_{1} \times N_{2}, H^{2}(N, V)$ is 2-dimensional. If $n_{2}>4$, then $N$ is perfect, and $H^{1}(N, V)=0$. If $n_{2}=4$, then $H^{1}(N, V)=\operatorname{Hom}(N, V)=0$. Thus, $\operatorname{dim} H^{2}(H, V) \leq 3$.

Suppose that $V$ is nontrivial. Let $W$ be an irreducible $F N$-submodule. Write $W=W_{1} \otimes W_{2}$ with the $W_{i}$ irreducible $N_{i}$-modules. If both of the $W_{i}$ are nontrivial, then $H^{1}(N, W)=0$ by Lemma 3.10 and so $H^{1}(N, V)=0$ (since $V$ is a direct sum of submodules of the form $g W, g \in H)$. Also by Lemma 3.10, $H^{2}(N, W)=$ $H^{1}\left(N_{1}, W_{1}\right) \otimes H^{1}\left(N_{2}, W_{2}\right)$ has dimension at most $(\operatorname{dim} W) / 4$ (we leave it to the reader to verify this when $\left.n_{2}=4\right)$. It follows by Lemma 3.8 that $\operatorname{dim} H^{2}(H, V) \leq$ $(\operatorname{dim} V) / 4$.

If $W_{1}$ is nontrivial, but $W_{2}$ is trivial, then $\operatorname{dim} H^{2}(N, W) \leq \operatorname{dim} H^{2}\left(N_{1}, W\right) \leq$ $(65 / 24) \operatorname{dim} W$. By Lemma 3.8,

$$
\begin{gathered}
\operatorname{dim} H^{2}(H, V) \leq \operatorname{dim} H^{2}\left(H / N, V^{N}\right)+\operatorname{dim} H^{2}(N, V)^{H} \\
+\operatorname{dim} H^{1}\left(H / N, H^{1}(N, V)\right) .
\end{gathered}
$$

Note that $V^{N}=0$ and as noted above the middle term is at most $(65 / 24)(\operatorname{dim} V)$. Finally, observe that since $H^{1}\left(N_{2}, F\right)=0, \operatorname{dim} H^{1}(N, V)=\operatorname{dim} H^{1}\left(N_{1}, V\right)<$ $\operatorname{dim} V / 8$ (this last inequality follows by Theorem 6.1 for $2^{a}>8$ ). This gives $\operatorname{dim} H^{2}(H, V) \leq(17 / 6) \operatorname{dim} V$.

Finally, suppose that $N_{1}$ is trivial on $V$, but $N_{2}$ is not. We consider the inequality above. The first term on the right side of the inequality is 0 . If $n_{2}>8$, then the middle term is at most $\operatorname{dim} H^{2}\left(N_{2}, V\right) \leq(65 / 24) \operatorname{dim} V$ and $\operatorname{dim} H^{1}(N, V)=$ $\operatorname{dim} H^{1}\left(N_{2}, V\right) \leq(1 / 13) \operatorname{dim} V$. Thus

$$
\operatorname{dim} H^{2}(H, V) \leq((65 / 24)+(1 / 13))(\operatorname{dim} V)<(17 / 6) \operatorname{dim} V .
$$

If $n_{2}<8$, it follows by [42] that $\operatorname{dim} H^{2}\left(N_{2}, V\right)+\operatorname{dim} H^{1}\left(N_{2}, V\right) \leq \operatorname{dim} V$ for all nontrivial irreducible modules. In particular, it follows that $\operatorname{dim} H^{2}(H, V) \leq$ $(17 / 6)(\operatorname{dim} V)$ for all values of $n_{2}$.

Arguing as usual, we see that the number of trivial composition factors of $N_{1}$ on $M$ is at most $(\operatorname{dim} M) / 2$. Thus, by the previous arguments, $\operatorname{dim} H^{2}(H, M) \leq$ $(3 / 2+17 / 12) \operatorname{dim} M=(35 / 12) \operatorname{dim} M$.

\section{SL: low rank}

In this section, we consider the groups $\operatorname{SL}(d, q), d \leq 4$. We use a gluing argument and the bounds for $\operatorname{SL}(4, q)$ and $S_{d}$ to get bounds for all $\operatorname{SL}(d, q)$ in the next section.

We start with an improvement of the bound given in (1.2) for $H^{1}$ in the natural characteristic. There are much better bounds for cross characteristic representations [28]. We will use Lemma 3.6 without comment below. 
Theorem 7.1. Let $G=\operatorname{SL}(d, q)$ be quasisimple and $F$ an algebraically closed field of characteristic $p$ with $q=p^{e}$. Let $M$ be an irreducible $F G$-module.

(1) Ifd $=2$, then $\operatorname{dim} H^{1}(G, M) \leq(\operatorname{dim} M) / 3$ unless either $p=2$ and $\operatorname{dim} M=$ 2 or $p=3$ and $G=\operatorname{SL}(2,9)$ with $\operatorname{dim} M=4$.

(2) $\operatorname{dim} H^{1}(G, M) \leq(\operatorname{dim} M) / d^{\prime}$, where $d^{\prime}$ is the largest prime with $d^{\prime} \leq d$.

Proof. If $d=2$, all first cohomology groups have been computed [1], and the result holds by inspection.

If the center of $G$ acts nontrivially on $M$, then $H^{1}(G, M)=0$ by Lemma 3.12. So we may view $M$ as an $H$-module with $H=\operatorname{PSL}(d, q)$. Note that by Lemma 3.8, $H^{1}(G, M) \cong H^{1}(H, M)$.

We now prove (2) when $d$ is an odd prime. Let $T$ be a maximal (irreducible) torus of size $\left(q^{d}-1\right) /((q-1) \operatorname{gcd}(d, q-1))$. Let $N=N_{H}(T)$ and note that $N / T$ is cyclic of order $d$. We claim that $d$ does not divide $|T|$. If $d$ does not divide $q-1$, this follows from the fact that $q^{d} \equiv q \bmod d$. If $d \mid(q-1)$, then write $q=1+d^{j} s$ where $d$ does not divide $s$. Then since $d$ is odd, $q^{d}=1+d^{j+1} s^{\prime}$ where $d$ does not divide $s^{\prime}$ and this proves the claim.

Let $y \in N$ be of order $d$. We claim that $C_{T}(y)=1$. Since $\operatorname{gcd}(|T|, d)=1$, we can lift $T$ to an isomorphic subgroup of $\operatorname{SL}(d, q)$ and it suffices to prove $C_{T}(y)=1$ in $\operatorname{SL}(d, q)$. Then we can identify $T$ with the norm 1 elements in $\mathbb{F}_{q^{d}}^{*}$ of order prime to $d$ and $y$ induces the $q$-Frobenius automorphism of this field, whence its fixed point set is $\mathbb{F}_{q}^{*}$. This has trivial intersection with $T$, whence the claim follows. This also implies that $y$ permutes all nontrivial characters of $T$ in orbits of size $d$. Thus $\operatorname{dim}[T, M]^{\langle y\rangle}=(\operatorname{dim}[T, M]) / d$.

Note that up to conjugacy, $y$ is a $d$-cycle in $S_{d} \leq \operatorname{PSL}(d, q)$. So $y$ is conjugate to $y^{-1}$ in $S_{d}$ and so also in $H$. So choose $z \in H$ that inverts $y$. Then $z$ does not normalize $T$ (since $y$ is not conjugate to $y^{-1}$ in $N$ ). It now follows by the main result of [9] (based on [24]) that $H=\left\langle N, z N z^{-1}\right\rangle$. Apply [23, Lemma 4] to conclude that $\operatorname{dim} H^{1}(H, M) \leq \operatorname{dim}[T, M]^{\langle y\rangle} \leq(\operatorname{dim} M) / d$.

We now complete the proof of (2) by induction. We have proved the result for $d$ any odd prime. It is more convenient work with $\mathbb{F}_{q} G$-modules. Suppose $d$ is not prime - in particular, $d^{\prime} \leq d-1$ in (2). Let $P$ be a maximal parabolic stabilizing a 1-space. So $P=L Q$ where $Q$ is the unipotent radical of $P, L$ is a Levi subgroup with $L \cong \operatorname{GL}(d-1, q)$ and $Q$ is the natural module for $J=\operatorname{SL}(d-1, q) \leq L$. Since $P$ contains a Sylow $p$-subgroup of $G$, it suffices to prove the bound for $H^{1}(P, V)$ with $V$ an irreducible $L$-module. If $V$ is not isomorphic to $Q$ as $\mathbb{F}_{p} L$-modules (equivalently, if $V$ is not isomorphic to a Galois twist of $Q$ as $\mathbb{F}_{q} L$-modules), then $H^{1}(P, V)=H^{1}(L, V)$ (by Lemma 3.8) and the result follows by induction. If $V \cong Q$, then $H^{1}(L, Q)=0$ unless $q=2$ and $d=4$ (cf. [7]). This implies that $\operatorname{dim} H^{1}(P, V)=1 \leq(\operatorname{dim} V) /(d-1) \leq(\operatorname{dim} V) / d^{\prime}$. If $q=2$ and $d=4$, then $G=A_{8}$, and the result is in [42]. 


\subsection{SL(2)}

Theorem 7.2. Let $G=\operatorname{SL}\left(2, p^{e}\right)$ with $p$ odd, $p^{e} \geq 5$ and $F$ an algebraically closed field of characteristic $r>0$. Let $M$ be an irreducible FG-module. Set $q=p^{e}$.

(1) If $r \neq 2$ and $r \neq p$, then $\operatorname{dim} H^{2}(G, M) \leq 2(\operatorname{dim} M) /(q-1)$.

(2) If $p \neq r=2$, then $\operatorname{dim} H^{2}(G, M) \leq \operatorname{dim} M$.

(3) If $r=p$, then $\operatorname{dim} H^{2}(G, M) \leq(\operatorname{dim} M) / 2$.

Proof. If $q \leq 11$, these results all follow by direct computation [42]. So assume that $q>11$.

In (1), a Sylow $r$-subgroup of $G$ is cyclic, whence Lemma 3.5 implies that $\operatorname{dim} H^{2}(G, M) \leq 1$. Since the smallest nontrivial representation of $\operatorname{SL}(2, q)$ in any characteristic other than $p$ has dimension $(q-1) / 2$, (1) holds.

Now consider (2). We first bound $\operatorname{dim} H^{2}(\operatorname{PSL}(2, q), M)$. Set $H=\operatorname{PSL}(2, q)$. We refer the reader to [11] for basic facts about the 2-modular representations of $H$. In particular, every irreducible representation is the reduction of an irreducible representation in characteristic zero. The characters of these representations are described in [14, Theorem 38.1]. Let $B=T U$ be a Borel subgroup of $H$ (of order $q(q-1) / 2$ ) with $T$ a torus of order $(q-1) / 2$. Note that $B$ has a normal subgroup $U T_{0}$ of index a power of $r$.

It follows from [11] and [14, 38.1] that either $M$ is one of two Weil modules of dimension $(q-1) / 2$ or has dimension $q \pm 1$. Moreover, it follows that the modules of dimension $q+1$ are all of the form $\lambda_{B}^{H}$ with $\lambda$ a nontrivial 1-dimensional character of $B$, and so by Shapiro's Lemma, $H^{2}(H, M) \cong H^{2}(B, \lambda)$. Since $\lambda$ is nontrivial, it is nontrivial on $U T_{0}$ and so by Lemma 3.12, $H^{2}(B, \lambda)=0$.

Suppose that $\operatorname{dim} M=q-1$. If $4 \mid(q-1)$, then $M$ is projective (cf. [14, 62.3, 62.5]) and so $H^{2}(H, M)=0$. So suppose that $4 \mid(q+1)$. By inspection of the character tables in $[14,38.1], M$ is multiplicity free as a $U$-module and so every nontrivial character of $U$ occurs precisely once in $M$. Since $T$ acts semiregularly on the nontrivial characters of $U$ (and so all the $U$-eigenspaces as well), $M$ is a free rank 2 module for the split torus $T$. Let $x$ be an involution inverting $T$. Note that $|T|=(q-1) / 2$ is odd. Thus, the only $T$-eigenspace that is $x$-invariant is the trivial eigenspace. Write $M=[T, M] \oplus M^{T}$. So all Jordan blocks of $x$ on $[T, M]$ are of size 2. Since $\operatorname{dim} M^{T}=2, x$ has either 0 or 2 Jordan blocks of size 1. Since there is a unique class of involutions in $H$, this implies that if $Y$ is any cyclic 2-subgroup of $H$, then $Y$ has at most 2 Jordan blocks of less than maximal size on $M$. Since Jordan blocks of maximal size correspond to projective modules, this implies that $\operatorname{dim} H^{k}(Y, M) \leq 2$ for $k>0$.

Let $J$ be a nonsplit torus of order $(q+1) / 2$. There is a unique class of involutions in $H$ and so by conjugating we may assume that $x$ is the unique involution in $J$. Write $J=J_{1} \times J_{2}$ where $J_{1}$ has odd order and $J_{2}$ is the Sylow 2-subgroup of $J$. 
Set $s=\left|J_{2}\right|$. We see from [11] and [14] that $\operatorname{dim} M^{J_{1}}=2 s$ or $2 s-2$ and so from our observations about the Jordan structure of the involution in $J, J$ has at most 3 Jordan blocks with trivial character and at most 2 of those have size less than $s$. By Lemma 3.12, $H^{j}\left(J,\left[J_{1}, M\right]\right)=0$. So $H^{j}(J, M)=H^{j}\left(J_{2}, M^{J_{1}}\right)$. Thus, $\operatorname{dim} H^{j}(J, M) \leq 2$ for $j>0$ and $\operatorname{dim} M^{J} \leq 3$. Let $L=N_{H}(J)$ and note that $[H: L]$ is odd. By Lemma 3.8,

$$
\begin{gathered}
\operatorname{dim} H^{2}(L, M) \leq \operatorname{dim} H^{2}\left(L / J, M^{J}\right)+\operatorname{dim} H^{2}(J, M) \\
+\operatorname{dim} H^{1}\left(L / J, H^{1}(J, M)\right) .
\end{gathered}
$$

The first term on the right is at most 3, the middle term at most 2 and the last term at most 2. Thus, $\operatorname{dim} H^{2}(G, M) \leq 7$. Since $q>11$, this implies that $\operatorname{dim} H^{2}(G, M)<$ $(7 / 18) \operatorname{dim} M<(\operatorname{dim} M) / 2$.

Finally, consider the case that $M$ is a Weil module. In this case (by [14, Theorem 38.1]), $Q$ has $(q-1) / 2$ distinct characters on $M$ that are freely permuted by $T$, whence $M \cong F T$ as an $F T$-module. If $4 \mid(q-1)$, then the normalizer of $T$ has odd index, and arguing as above, we see that $\operatorname{dim} H^{2}(H, M) \leq 1$. If 4 does not divide $q-1$, then as above, we see that an involution has precisely one trivial Jordan block, and so if $i>0, \operatorname{dim} H^{i}(J, M) \leq 1$. In all cases $\operatorname{dim} H^{2}(H, M) \leq 3$. Thus, $\operatorname{dim} H^{2}(H, M)<(\operatorname{dim} M) / 2$ (since $\left.q \geq 11\right)$.

Let $Z=Z(G)$ and note that $|Z|=2$. In all cases, $\operatorname{dim} H^{2}(G, M) \leq \operatorname{dim} H^{2}(H, M)+\operatorname{dim} H^{2}(Z, M)^{G}+\operatorname{dim} H^{1}\left(H, H^{1}(Z, M)\right)$. If $M$ is trivial, then $\operatorname{dim} H^{2}(G, M)=1$. Otherwise, the middle term on the right is 0 . The first term on the right is at most $\operatorname{dim} M / 2$. The last term is at most $(\operatorname{dim} M) / 2$. Thus, $\operatorname{dim} H^{2}(G, M) \leq \operatorname{dim} M$.

Finally, consider (3). It is more convenient to work over $F=\mathbb{F}_{p}$ in this case. Let $B=T U$ be a Borel subgroup with $|U|=q$.

Let $W$ be an irreducible $F B$-module. Then by Lemma 3.8 and the fact that $T$ has order coprime to $p$,

$$
\operatorname{dim} H^{2}(B, W) \leq \operatorname{dim} H^{2}(U, W)^{T} .
$$

Note that $U$ is a $T$-module by conjugation. As we have seen (Lemma 3.16), $H^{2}(U, W)=\wedge^{2}\left(U^{*}\right) \otimes W \oplus U^{*} \otimes W$. So $T$ has fixed points if and only if either $U \cong W$ or $W$ is a homomorphic image of $\wedge^{2}(U)$. Note we are taking exterior powers over $F$ and so $\wedge^{2}(U)$ has dimension $e(e-1) / 2$. Suppose that $\alpha$ of order $(q-1) / 2$ is an eigenvalue on $U$ for a generator $t$ of $T$. Then the eigenvalues of $t$ on $U$ (over the algebraic closure) are just the $e$ Galois conjugates of $\alpha$. So the eigenvalues of $t$ on $\wedge^{2}(U)$ are all Galois conjugates of $\alpha^{1+p^{j}}$ for some $0<j<e$. Note that this is never a Galois conjugate of $\alpha$ and also $T$ has no multiple eigenvalues on $\wedge^{2}(U)$ (by Lemma 3.17). 
So we have seen that $H^{2}(B, W)=0$ unless $W \cong U$ or $W \cong U \otimes_{\mathbb{F}_{q}} U^{p^{j}}$ for some $j$ with $1<j<e$ as $\mathbb{F}_{p} T$-modules. Moreover, as noted above, $\wedge^{2}(U)$ is multiplicity free, and so, using Lemma 3.16, $\operatorname{dim} H^{2}(B, W)^{T} \leq \operatorname{dim} W$.

This already gives the inequality $\operatorname{dim} H^{2}(G, M) \leq \operatorname{dim} H^{2}(B, M) \leq \operatorname{dim} M$. If $M$ is trivial, then $H^{2}(G, M)=0$. If $M$ is irreducible and nontrivial, then if $W$ occurs as a $T$-submodule, so does $W^{*}$. Thus, in fact, $\operatorname{dim} H^{2}(B, M) \leq(\operatorname{dim} M) / 2$.

Theorem 7.3. Let $G=\operatorname{SL}(2, q)$ with $q=2^{e} \geq 4$, and let $F$ be an algebraically closed field of characteristic $r>0$. Let $M$ be an irreducible FG-module.

(1) If $r \neq 2$, then $\operatorname{dim} H^{2}(G, M) \leq(\operatorname{dim} M) /(q-1)$.

(2) If $r=2$, then $\operatorname{dim} H^{2}(G, M) \leq(\operatorname{dim} M) / 2$ unless $2^{e}=4$ and $M$ is the trivial module.

Proof. If $r$ is odd, then a Sylow $r$-subgroup is cyclic, whence $\operatorname{dim} H^{2}(G, M) \leq 1$ by Lemma 3.5. If $M$ is trivial, $H^{2}(G, M)=0$. It is obvious that the smallest faithful representation for a Borel subgroup has dimension $q-1$, whence also for $G$.

Let $r=2$. If $q>4$, the proof is identical to the proof in the previous lemma when $p=r$. If $q=4$, then $\wedge^{2}(U)$ is the trivial module, which explains why $H^{2}(\operatorname{SL}(2,4), F) \neq 0$. For $q=4$, the result follows by [42].

\section{2. $\operatorname{SL}(3)$}

Theorem 7.4. Let $p$ and $r$ be primes. Let $G=\operatorname{SL}(3, q), q=p^{e}, F=\mathbb{F}_{r}$ and $M$ an irreducible $F G$-module. Then either $\operatorname{dim} H^{2}(G, M) \leq \operatorname{dim} M$ or $3=r \neq p$ and $\operatorname{dim} H^{2}(G, M) \leq(3 / 2) \operatorname{dim} M$.

Proof. If $q \leq 4$, the result follows by a direct computation [42]. So assume that $q>4$.

First consider the case that $r \neq p$.

If $r$ does not divide $q-1$, then a Sylow $r$-subgroup of $G$ is cyclic, whence the result holds by Lemma 3.5 .

Next suppose that $3 \neq r \mid(q-1)$. Then a Sylow $r$-subgroup fixes a 1-space and a complementary 2 -space in the natural representation. Let $P$ be the full stabilizer of one of these subspaces with unipotent radical $Q$. Then for one of the choices for $P, \operatorname{dim} M^{Q} \leq(\operatorname{dim} M) / 2$. Note that $P=L Q$ with $L=\operatorname{GL}(2, q)$. Let $J=\operatorname{SL}(2, q)<L$. So $\operatorname{dim} H^{2}(G, M) \leq \operatorname{dim} H^{2}(P, M) \leq \operatorname{dim} H^{2}\left(L, M^{Q}\right)$ (here we are using Lemma 3.8 and the fact that $r$ does not divide $|Q|$ ). Let $V$ be an irreducible $L$-module. If $V$ is the trivial module, then $\operatorname{dim} H^{2}(L, V)=1$ (since $J$ has trivial Schur multiplier and $L / J$ is cyclic of order a multiple of $r$ ). Otherwise, by Lemma 3.8,

$$
\operatorname{dim} H^{2}(L, V) \leq \operatorname{dim} H^{2}(J, V)+\operatorname{dim} H^{1}\left(L / J, H^{1}(J, V)\right) .
$$


By the results of the previous subsection we have $\operatorname{dim} H^{2}(J, V) \leq \operatorname{dim} V$, and by $(1.2) \operatorname{dim} H^{1}(J, V) \leq(\operatorname{dim} V) / 2$. Thus, $\operatorname{dim} H^{2}(G, M) \leq(1.5) \operatorname{dim} M^{Q} \leq$ (3/4) $\operatorname{dim} M$.

Suppose that $r=3$ does divide $q-1$. Then a Sylow 3-subgroup is contained in the normalizer $H$ of a split torus $T$ and $H / T \cong S_{3}$.

Let $V$ be an irreducible $F H$-module. If $T$ is nontrivial on $V$, then by Corollary $3.12, H^{2}(H, V)=0$. So we may assume that $\operatorname{dim} V=1$ and $H$ either acts trivially on $V$ or via the sign representation for $S_{3}$. Now we use Lemma 3.8 to see that

$$
\operatorname{dim} H^{2}(H, V) \leq \operatorname{dim} H^{2}\left(S_{3}, V\right)+\operatorname{dim} H^{2}(T, V)^{H}+\operatorname{dim} H^{1}\left(S_{3}, H^{1}(T, V)\right) .
$$

Note that $H^{1}(T, V) \cong \operatorname{Hom}(T, V)$ is an indecomposable 2-dimensional $S_{3}$ module. Thus, by Lemma 3.5, the left and right hand terms of the right side of the above inequality are each at most 1 . Finally, by Lemma 3.16, there is an exact sequence

$$
0 \rightarrow \operatorname{Hom}_{H}(T, V) \rightarrow H^{2}(T, V)^{H} \rightarrow \operatorname{Hom}_{H}\left(\wedge^{2}(T), V\right) .
$$

Note that the only $H$-simple homomorphic image of $T$ is the sign representation for $S_{3}$ while $\wedge^{2}(T)$ only surjects onto the trivial module. Thus, $\operatorname{dim} H^{2}(T, V)^{H} \leq 1$ and so $\operatorname{dim} H^{2}(H, V) \leq 3 \operatorname{dim} V$. Let $T_{0}$ denote the Hall $3^{\prime}$-subgroup of $T$. Then $M=\left[T_{0}, M\right] \oplus M^{T_{0}}$. Since $q>4, T_{0}$ is nontrivial (indeed it is either a Klein group of order 4 or contains a regular semisimple element). Considering the maximal subgroups of SL $(3, q)$ [40] and [27], there are two conjugates of $T_{0}$ which generate $G$, whence $\operatorname{dim} M^{T_{0}} \leq(\operatorname{dim} M) / 2$ by Lemma 3.19. Since $H^{2}\left(H,\left[T_{0}, M\right]\right)=0$ by Lemma 3.12, the computation above shows that $\operatorname{dim} H^{2}(H, M) \leq 3 \operatorname{dim} M^{T_{0}} \leq$ $(3 / 2)(\operatorname{dim} M)$.

Now consider the case $p=r$. Let $P=L Q$ be the stabilizer of a 1-space or a hyperplane where $Q$ is the unipotent radical of $P$ and $L \cong \operatorname{GL}(2, q)$ is a Levi complement. Let $Z=Z(L)$ and note that $Z$ is cyclic of order $q-1$. Let $T$ be a split torus containing $Z$ (of order $(q-1)^{2}$ ).

Let $V$ be an irreducible $F P$-module. It suffices to prove that $\operatorname{dim} H^{2}(P, V) \leq$ $\operatorname{dim} V$. By Lemma 3.8,

$$
\operatorname{dim} H^{2}(P, V) \leq \operatorname{dim} H^{2}(L, V)+\operatorname{dim} H^{2}(Q, V)^{L}+\operatorname{dim} H^{1}\left(L, H^{1}(Q, V)\right) .
$$

Consider the middle term on the right. Using Lemma 3.16 and arguing as in the proof of Lemma 3.17, $\wedge^{2} Q$ has distinct composition factors as an $L$-module (and no composition factor is isomorphic to $Q$ as an $L$-module), whence the second term has dimension at most $(\operatorname{dim} V) / 2$ (since the dimension of $V$ is at least 2 over $\operatorname{End}_{G}(V)$ unless $[L, L]$ acts trivially on $V$ but then $V$ is not a homomorphic image of $Q$ or $\left.\wedge^{2}(Q)\right)$.

Suppose that $Z$ is trivial on $V$. Since $q>4, Z$ is nontrivial on $Q$ and every composition factor of $\wedge^{2} Q$, whence the middle term on the right is 0 . Similarly, $Z$ acts 
without fixed points on $H^{1}(Q, V)$ and so by Corollary 3.12, $H^{1}\left(L, H^{1}(Q, V)\right)=0$ as well. Thus, $\operatorname{dim} H^{2}(P, V) \leq \operatorname{dim} H^{2}(L, V)$ and this is at most $(\operatorname{dim} V) / 2$ by the result for SL(2).

Now suppose that $Z$ is nontrivial on $V$. By Lemma 3.12, $H^{2}(L, V)=0$. Note that $W:=H^{1}(Q, V) \cong Q^{*} \otimes V$ as an $L$-module (however, the tensor product is over $\left.\mathbb{F}_{p}\right)$. Since $V$ is $L$-irreducible, it must be $Z$-homogeneous. By Lemma 3.12, $H^{1}(L, W)=H^{1}\left(L, W^{Z}\right)$ and this is either 0 unless $V$ and $Q$ involve the same $\mathbb{F}_{p} Z$-irreducible module. If that is the case, then $\operatorname{dim} W^{Z}=2 \operatorname{dim} V$. Thus by (1.2), $\operatorname{dim} H^{1}(L, W) \leq \operatorname{dim} V$. In this case, $\operatorname{Hom}_{Z}\left(\wedge^{2} Q, V\right)=0$ and so $\operatorname{dim} H^{2}(P, V) \leq$ $\operatorname{dim} V$ unless perhaps $Q \cong V$. We still obtain this inequality since we compute in this case that $\operatorname{dim} H^{1}(L, W)<\operatorname{dim} V$.

If $Z$ is nontrivial on $V$, but $V$ and $Q$ do not involve the same irreducible $\mathbb{F}_{p} Z$ module, then $H^{1}(L, W)=0$ and so in this case $\operatorname{dim} H^{2}(P, V) \leq(\operatorname{dim} V) / 2$.

\subsection{SL(4)}

Theorem 7.5. Let $G=\operatorname{SL}(4, q), q=p^{e}$. Let $F=\mathbb{F}_{p}$. If $M$ is an irreducible $\mathbb{F}_{p} G$-module, then $\operatorname{dim} H^{2}(G, M)<2 \operatorname{dim} M$.

Proof. If $q \leq 3$, see [42]. So assume that $q>3$. Let $P$ be the stabilizer of a 1 -space. Since $P$ contains a Sylow $p$-subgroup, it suffices to bound $\operatorname{dim} H^{2}(P, M)$. Write $P=L Q$ where $Q$ is the unipotent radical of $P$ and $L \cong \operatorname{GL}(3, q)$ is the Levi complement.

By Lemma 3.8

$\operatorname{dim} H^{2}(P, M) \leq \operatorname{dim} H^{2}\left(L, M^{Q}\right)+\operatorname{dim} H^{2}(Q, M)^{L}+\operatorname{dim} H^{1}\left(L, H^{1}(Q, M)\right)$.

Since $G$ is generated by $Q$ and the radical of the opposite parabolic, by replacing $P$ by its opposite, we may assume that $\operatorname{dim} M^{Q} \leq(1 / 2)(\operatorname{dim} M)$, and so by the result for $\operatorname{SL}(3), \operatorname{dim} H^{2}\left(L, M^{Q}\right) \leq(1 / 2) \operatorname{dim} M$.

Consider $H^{2}(Q, M)$ as an $L$-module. By taking a $P$-composition series for $M$, it suffices to bound $\operatorname{dim} H^{2}(Q, V)^{L}$ where $V$ is an irreducible $F P$-module. By Lemma 3.16 we have the exact sequence,

$$
0 \rightarrow \operatorname{Hom}(Q, V)^{L} \rightarrow H^{2}(Q, V)^{L} \rightarrow \operatorname{Hom}\left(\wedge^{2}(Q), V\right)^{L}
$$

Since $Q$ is an irreducible $F L$-module, the second term either is zero or is isomorphic to $\operatorname{End}_{L}(Q) \cong \mathbb{F}_{q}$ and so has dimension $(\operatorname{dim} V) / 3$. Next consider $\wedge^{2}(Q)$ over $\mathbb{F}_{p}$. Note that $Q \otimes_{\mathbb{F}_{p}} \mathbb{F}_{q}$ is the sum of Galois twists $Q_{i}, 1 \leq i \leq e$ as an $\mathbb{F}_{q} L$-module. The exterior square will be the sum of all $Q_{i} \otimes Q_{j}, i<j$ plus the sum of all $\wedge^{2} Q_{i}$. We note that these are all irreducible and nonisomorphic as $\mathbb{F}_{q} L$-modules. Since none of them is isomorphic to $Q$, it follows that if $V=Q$, then $\operatorname{dim} H^{2}(Q, V)^{L} \leq(\operatorname{dim} V) / 3$. So assume this is not the case. Thus, 
$\operatorname{Hom}\left(\wedge^{2}(Q), V\right)^{L}=0$ unless $V$ is isomorphic to one of $Q_{i} \otimes Q_{j}$ or $\wedge^{2}\left(Q_{i}\right)$ If $V$ is one of these modules, then $\operatorname{Hom}\left(\wedge^{2}(Q), V\right)^{L} \cong \operatorname{End}_{L}(V)$ and so has dimension at most $(\operatorname{dim} V) / 3$. Thus, $H^{2}(Q, V)^{L}$ has dimension at most $(\operatorname{dim} V) / 3$.

Finally, consider the far right term of the sequence above. Again, we can take a $P$-composition series for $M$ and consider $H^{1}\left(L, H^{1}(Q, V)\right)$ for $V$ an irreducible $F P$-module. Now $H^{1}(Q, V)=\operatorname{Hom}(Q, V)\left(\right.$ over $\left.\mathbb{F}_{p}\right)$. Let $T=Z(L)$. By Corollary 3.12, $H^{1}\left(L, H^{1}(Q, V)\right)=H^{1}\left(L, H^{1}(Q, V)^{T}\right)$. Then $\operatorname{dim} H^{1}(Q, V)^{T} \leq$ $3 \operatorname{dim} V$. So applying Theorem 7.1, we see that $\operatorname{dim} H^{1}\left(L, H^{1}(Q, V)\right) \leq \operatorname{dim} V$. Thus, $\operatorname{dim} H^{2}(G, M) / \operatorname{dim} M<1 / 2+1 / 3+1<2$ as required.

Theorem 7.6. Let $G=\mathrm{SL}(4, q), q=p^{e}$. Let $F=\mathbb{F}_{r}$ for $r$ a prime. If $M$ is an irreducible $F G$-module, then $\operatorname{dim} H^{2}(G, M)<2 \operatorname{dim} M$.

Proof. By the previous result we may assume that $r \neq p$.

Let $R$ be a Sylow $r$-subgroup. We consider various cases.

First suppose that $r>3$, whence $R$ is abelian. If $r \mid(q-1)$, then $R \leq J$, the monomial group $J:=T . S_{4}$ where $T$ is a split torus. Since $r$ does not divide $\left|S_{4}\right|$, Lemma 3.8 and Corollary 3.12 imply that $\operatorname{dim} H^{2}(J, M) \leq \operatorname{dim} H^{2}(T, M)^{S_{4}}$. It suffices to prove the inequality for $W$ irreducible for $J$. If $T$ is not trivial on $W$, then $H^{2}(J, W)=0$. If $T$ is trivial on $W$, then by Lemma 3.16, $\operatorname{dim} H^{2}(T, W) \leq$ $\operatorname{dim} \operatorname{Hom}_{G}(T, W)+\operatorname{dim} \operatorname{Hom}_{G}\left(\wedge^{2}(T), W\right)$. Note that the only irreducible quotient of $T$ is the 3-dimensional summand of the permutation module. Similarly, the only irreducible quotient of $\wedge^{2}(T)$ is the same module. So if $W$ is not that module, $H^{2}(J, W)=0$. If $W$ is that module, then each term on the right in the above inequality is 1 and so $\operatorname{dim} H^{2}(J, W) \leq 2<3=\operatorname{dim} W$.

If $r>3$ and does not divide $q-1$, then $R$ has rank at most 2. If $R$ is cyclic, the result holds by Lemma 3.5. If not, then $R$ is contained in the stabilizer of a 2 -space. The radical $Q$ of this parabolic has fixed space of dimension at most $(\operatorname{dim} M) / 2$ (since $Q$ and its opposite generate $G)$. Lemma 3.8 together with the fact that $\operatorname{dim} H^{2}(R, F)=3$ gives $\operatorname{dim} H^{2}(R, M) \leq(3 / 2)(\operatorname{dim} M)$, and the result holds.

Suppose $r=3$. If 3 does not divide $q-1$, then $R$ is abelian and stabilizes a 2-space and the argument above applies. So suppose that $3 \mid(q-1)$. Then $R$ fixes a 1-space and 3-space, and so is contained in the corresponding parabolic $P=Q L$. We may assume that $\operatorname{dim} M^{Q} \leq(\operatorname{dim} M) / 2$. Thus, $\operatorname{dim} H^{2}(P, M) \leq \operatorname{dim} H^{2}\left(L, M^{Q}\right)$.

By the result for $\operatorname{SL}(3, q)$ (Theorem 7.4), we see that $H^{2}\left(J, M^{Q}\right)$ has dimension at most $(3 / 2)\left(\operatorname{dim} M^{Q}\right)$ where $J$ is the derived subgroup of $L$. So

$$
\begin{gathered}
\operatorname{dim} H^{2}\left(L, M^{Q}\right) \leq \operatorname{dim} H^{2}\left(L / J, M^{Q L}\right)+\operatorname{dim} H^{2}\left(J, M^{Q}\right) \\
+\operatorname{dim} H^{1}\left(L / J, H^{1}\left(J, M^{Q}\right)\right) .
\end{gathered}
$$

The terms on the right are bounded by $(\operatorname{dim} M) / 2,(3 / 4) \operatorname{dim} M$ and $(\operatorname{dim} M) / 4$, whence $\operatorname{dim} H^{2}(G, M)<2 \operatorname{dim} M$. 
Finally, consider the case $r=2$. If $q=3$, see [42]. So assume that $q \geq 5$. We work over an algebraically closed field. If $M$ is the trivial module, then $\operatorname{dim} H^{2}(G, F)=0$ (since the Schur multiplier of $\operatorname{SL}(4, q)$ is trivial). So assume that $M$ is not trivial. By computing orders, we see that $R$ is contained in $H$, the stabilizer of a pair of complementary 2-spaces. Let $L=L_{1} \times L_{2}=\operatorname{SL}(2, q) \times \operatorname{SL}(2, q)$. Note that $L$ is normal in $H$, and $H / L$ is a dihedral group of order $2(q-1)$.

Let $V$ be an irreducible $H$-module. If $V^{L}=0$, let $W$ be an irreducible $L$-submodule of $V$. So $W=W_{1} \otimes W_{2}$ with $W_{i}$ an irreducible $L_{i}$-module. If each $W_{i}$ is nontrivial, then by Lemma 3.10, $H^{1}(L, V)=0$, and so by Lemma 3.11, it follows that $\operatorname{dim} H^{2}(H, V) \leq \operatorname{dim} H^{2}(L, V) \leq(\operatorname{dim} V) / 4$. If $W_{1}$ is nontrivial and $W_{2}$ is trivial, then $V$ is an induced module, and so $H^{2}(H, V) \cong H^{2}(X, D)$ where $X / L$ is cyclic and $L_{2}$ is trivial on $D$. Then by Lemma 3.8,

$$
\operatorname{dim} H^{2}(X, D) \leq \operatorname{dim} H^{2}(L, D)+\operatorname{dim} H^{1}\left(X / L, H^{1}(L, D)\right) .
$$

Then $\operatorname{dim} H^{2}(L, D)=\operatorname{dim} H^{2}\left(L_{1}, D\right) \leq \operatorname{dim} D \leq(\operatorname{dim} V) / 2$. Furthermore, we know that $\operatorname{dim} H^{1}\left(X / L, H^{1}(L, D)\right) \leq \operatorname{dim} H^{1}(L, D)=\operatorname{dim} H^{1}\left(L_{1}, D\right)$. By $(1.2), \operatorname{dim} H^{1}\left(L_{1}, D\right) \leq(\operatorname{dim} D) / 2 \leq(\operatorname{dim} V) / 4$. Thus, $\operatorname{dim} H^{2}(H, V) \leq$ $(3 / 4)(\operatorname{dim} V)$.

If $V$ is trivial for $L$, then by Lemma 3.8, $\operatorname{dim} H^{2}(H, V) \leq \operatorname{dim} H^{2}(H / L, V)+$ $\operatorname{dim} H^{2}(L, V)+\operatorname{dim} H^{1}\left(H / L, H^{1}(L, V)\right)$. Since $L$ is perfect and since the Schur multiplier of $L$ is trivial, it follows by Lemma 3.9 that $\operatorname{dim} H^{2}(H, V) \leq$ $\operatorname{dim} H^{2}(H / L, V)$. Since $H / L$ is dihedral, by Corollary 3.12 it follows that either $H^{2}(H / L, V)=0$ or $V$ is trivial. It is easy to see that $H^{2}\left(H / L, \mathbb{F}_{2}\right)$ is 3 -dimensional. It is straightforward to see that $G$ can be generated by two conjugates of an odd order subgroup of $L$, whence $H$ can have at most (dim $M) / 2$ trivial composition factors by Lemma 3.19. Thus, $\operatorname{dim} H^{2}(H, M) / \operatorname{dim} M \leq(3 / 2)+(3 / 8)<2$.

\section{SL: the general case}

We handle $\operatorname{SL}(n)$ by means of a gluing argument. This is a variation of the presentations given in [21] and [22]. Note also that the proposition below applies in either the profinite or discrete categories. The key idea is that it suffices to check relations on subgroups generated by pairs of simple root subgroups - this is a consequence of the Curtis-Steinberg-Tits presentation (see [13]). We will also use this method to deduce the result for groups of rank at least 3 from the results on alternating groups and on rank 2 groups.

We state the Curtis-Steinberg-Tits result in the following form:

Lemma 8.1. Let $G$ be the universal Chevalley group of a given type of rank at least 2 over a given field. Let $\Pi$ be the set of simple positive roots of the corresponding Dynkin 
diagram and let $L_{\delta}$ be the rank one subgroup of $G$ generated by the root subgroups $U_{\delta}$ and $U_{-\delta}$ for $\delta \in \Pi$. Let $X$ be a group generated by subgroups $X_{\delta}, \delta \in \Pi$. Suppose that $\pi: X \rightarrow G$ is a homomorphism such that $\pi\left(X_{\delta}\right)=L_{\delta}$ and $\pi$ is injective on $\left\langle X_{\alpha}, X_{\beta}\right\rangle$ for each $\alpha, \beta \in \Pi$. Then $\pi$ is an isomorphism.

Let $X$ and $Y$ be two disjoint sets of size 2. Set $G=\operatorname{SL}(n, q)=\operatorname{SL}(V)$ for $n>4$. Let $e_{1}, \ldots, e_{n}$ be a basis for $V$. Let $\langle X \mid R\rangle$ be a presentation for $A_{n}$ (acting on the set $\{1, \ldots, n\})$ and $\langle Y \mid S\rangle$ a presentation for $\operatorname{SL}(4, q)$ acting on a space $W$ that is the span of $e_{1}, \ldots, e_{4}$ (viewing these either as profinite presentations or discrete presentations).

Let $G_{1}$ be the subgroup of $G$ consisting of the elements which permute the elements of the basis as even permutations. Let $G_{2}$ be the subgroup of $G$ that acts trivially on $e_{j}, j>4$ and preserves the subspace generated by $e_{1}, \ldots, e_{4}$. Let $L$ be the subgroup of $\operatorname{SL}(4, q)$ leaving the span of $\left\{e_{1}, e_{2}\right\}$ invariant and acting trivially on $e_{3}$ and $e_{4}$. So $L \cong \operatorname{SL}(2, q)$. Let $S \cong A_{4}$ be the subgroup of $\operatorname{SL}(4, q)$ consisting of the even permutations of $e_{1}, \ldots, e_{4}$. Pick generators $u, v$ of $S$ where $u=\left(e_{1} e_{2}\right)\left(e_{3} e_{4}\right)$ and $v=\left(\begin{array}{lll}e_{1} & e_{2} & e_{3}\end{array}\right)$. Note that $u$ normalizes $L$. Choose $a \in L$ such that $L=\left\langle a, a^{u}\right\rangle$ (e.g., we can take $a$ to be almost any element of order $q+1$ ).

Let $T \cong A_{4}$ be the subgroup of $A_{n}$ fixing all $j>4$. In $T$, let $u^{\prime}=(12)$ (34) and $v^{\prime}=(123)$. Let $K \cong A_{n-2}$ be the subgroup of $A_{n}$ fixing the first two basis vectors. Let $b$ and $c$ be any generators for $K$.

Let $J$ be the group generated by $X \cup Y$ with relations $R, S, u=u^{\prime}, v=v^{\prime}$, $[a, b]=[a, c]=1$. Let $J_{1} \leq J$ be the subgroup generated by $X$, and $J_{2}$ the subgroup generated by $Y$.

There clearly is a homomorphism $\gamma: J \rightarrow G$ determined by sending $J_{i}$ to $G_{i}$ for $i=1,2$ (where we send $X$ to the corresponding permutation matrices in $G$ and $Y$ to the corresponding elements in $G_{2}$ - all relations in $J$ are satisfied and so this gives the desired homomorphism). In particular, this shows that $J_{i} \cong G_{i}$ for $i=1$ and 2 and so we may identify $G_{i}$ and $J_{i}$. In particular, $u$ and $v$ are words in $Y$ and $u^{\prime}, v^{\prime}$ are words in $X$.

Proposition 8.2. $J \cong G$.

Proof. As we noted above, there is a surjection $\gamma: J \rightarrow G$ that sends $J_{1}$ to $G_{1}$ and $J_{2}$ to $G_{2}$. It suffices to show that $\gamma$ is an isomorphism. We also view $a, b$ and $c$ as elements of $J$, and $L$ as a subgroup of $J$.

We first show that $[K, L]=1$ in $J$. By the relations, we have that $[a, K]=1$. Since $u^{\prime}$ normalizes $K$ and $u=u^{\prime}$, we see that $1=\left[a^{u}, K^{u^{\prime}}\right]=\left[a^{u}, K\right]$. Since $L=\left\langle a, a^{u}\right\rangle,[K, L]=1$. Set $E:=\left\langle K, u^{\prime}\right\rangle \cong S_{n-2} \leq A_{n}$. Since $u$ normalizes $L$, we see that $E$ does as well. Note that $E$ is precisely the stabilizer in $A_{n}$ of the subset $\{1,2\}$. This is a maximal subgroup of $A_{n}$, and since $A_{n}$ does not normalize $L$ (since $\gamma\left(A_{n}\right)$ does not normalize $\gamma(L)$ in $G$ ), it follows that $E=N_{A_{n}}(L)$ (in $J$ ). 
Let $\Omega$ be the set of conjugates of $L$ under $A_{n}$ in $J$. By the previous remarks, $|\Omega|=$ $\left[A_{n}: S_{n-2}\right]=n(n-1) / 2$ and moreover, there is an identification between $\Omega$ and the subsets of size 2 of $\{1, \ldots, n\}$. Let $L_{i, j}$ denote the conjugate of $L$ corresponding to the subset $\{i, j\}$. Note that $\gamma\left(L_{i, j}\right)$ is the subgroup of $G$ that preserves the 2 -space $\left\{e_{i}, e_{j}\right\}$ and acts trivially on the other basis vectors of $V$.

Let $\Delta$ be the orbit of $L$ under $A_{4}$. Note that $|\Delta|=6$ and $\Delta$ corresponds to the two element subsets of $\{1,2,3,4\}$. Since $A_{n}$ is a rank 3 permutation group on $\Omega$, any pair of distinct conjugates of $L$ in $\Omega$ is conjugate to either the pair $\left\{L, L_{2,3}\right\}$ or $\left\{L, L_{3,4}\right\}$.

Suppose that $L_{1}$ and $L_{2}$ are two of these conjugates. By the above remarks, they are conjugate by some element in the group to $L$ and $M=L^{x}$ for some $x \in A_{4}$. In particular, we see that $M$ is the subgroup of $\operatorname{SL}(4, q)$ fixing the 2-space generated by $e_{x(1)}$ and $e_{x(2)}$ and fixing the vectors $e_{x(3)}$ and $e_{x(4)}$. Since we are now inside $\operatorname{SL}(4, q)$, we see that either $[L, M]=1$ or $L$ and $M$ generate an $\operatorname{SL}(3, q) \leq \operatorname{SL}(4, q)$. Since $\gamma$ is injective on $\operatorname{SL}(4, q), \gamma$ is injective on $\langle L, M\rangle$ and so is injective on the subgroup generated by $\left\langle L^{h_{1}}, L^{h_{2}}\right\rangle$ for any elements $h_{1}, h_{2} \in A_{n}$.

Therefore, by the Curtis-Steinberg-Tits relations (see Lemma 8.1), $N=\left\langle\left\{L^{g} \mid\right.\right.$ $\left.\left.g \in A_{n}\right\}\right\rangle \cong G$, and indeed $\gamma: N \rightarrow G$ is an isomorphism.

It suffices to show that $J=N$. Since $A_{n}$ normalizes $N$ and since $\operatorname{SL}(4, q) \leq N$ ( $\operatorname{SL}(4, q)$ contains the $A_{4}$ conjugates of $L$ and these generate $\operatorname{SL}(4, q)$ ), it follows that $N$ is normal in $J$. Clearly, $\operatorname{SL}(4, q)$ is trivial in $J / N$ and since $A_{n} \cap N \geq A_{4}$, it follows that $A_{n} \leq N$ as well. Thus, $J=N$ and the proof is complete.

Since $L$ and $K$ are 2-generated, $J$ is presented by 4 generators and $|R|+|S|+4$ relations.

By Theorem 6.2, we have profinite presentations for $A_{n}$ with 4 relations. By Theorems 7.5 and 7.6, $\mathrm{SL}(4, q)$ has a profinite presentation with 3 relations. Thus we have a profinite presentation for $\operatorname{SL}(n, q)$ with 4 generators and $4+3+4=11$ relations. Using Lemma 3.15, we obtain:

Corollary 8.3. Let $G=\operatorname{SL}(n, q)$ with $n \geq 5$. Then $G$ has a profinite presentation on 2 generators and 9 relations. In particular, $\hat{r}(G) \leq 9$.

Theorem 8.4. Let $G$ be a quasisimple group that surjects on $\operatorname{PSL}(n, q)$. Let $F$ be a field. Then

(1) $\hat{r}(G) \leq 9$; and

(2) $\operatorname{dim} H^{2}(G, M) \leq 8.5 \operatorname{dim} M$ for any $F G$-module $M$.

Proof. If $\operatorname{SL}(n, q)$ has trivial Schur multiplier, then (1) follows by Corollary 4.2 and the previous result. This is the case unless $(n, q)=(2,4),(2,9),(3,2),(3,4)$ or $(4,2)$ [16, p. 313]. In those cases, we have a smaller value for $\hat{r}(\operatorname{SL}(n, q))$ and Corollary 4.2 gives (1). Now (2) follows from (1) by (1.4). 


\section{Low rank groups}

In this section, we consider the rank one and rank two finite groups of Lie type. We also consider some of the rank three groups which are used for our gluing method.

The method for the low rank groups is fairly straightforward. With more work, one can obtain better bounds. As usual, we will use Lemma 3.6 without comment. We first consider the rank one groups.

Lemma 9.1. Let $G$ be the universal cover of a rank one simple finite group of Lie type.

(1) If $G=\operatorname{SL}(2, q)$, then $h(G) \leq 1$.

(2) If $G=S z(q), q=2^{2 k+1}>2$, then $h(G) \leq 1$.

(3) If $G=\mathrm{SU}(3, q), q>2$, then $h(G) \leq 2$.

(4) If $G=\mathfrak{R}(q), q=3^{2 k+1}>3$, then $h(G) \leq 3$.

Proof. Let $R$ be a Sylow $r$-subgroup of $G$ for some prime $r$. Let $F=\mathbb{F}_{r}$.

(1) is proved in the previous section and (2) is proved in [50].

Consider $G=\operatorname{SU}(3, q)$ with $q=p^{e}$. First suppose that $p \neq r$. If $r \neq 3$, then $R$ is either cyclic or stabilizes a nondegenerate subspace and so embeds in $\operatorname{GU}(2, q)$. We use the result for $\operatorname{SL}(2, q)$ and Lemma 3.8 to deduce the result.

If 3 does not divide $q+1$, the above argument applies to $r=3$. Suppose that $r=3 \mid(q+1)$. Then $R$ is contained in the stabilizer of an orthonormal basis and we argue precisely as we did for $\operatorname{SL}(3, q)$ in Theorem 7.4.

So assume that $p=r$ and $R \leq B$, a Borel subgroup. Write $B=T R$ with $T$ cyclic of order $q^{2}-1$. Let $Z=Z(R)$ of order $q$. If $q=4$, one computes directly that the bound holds. So assume that $q>4$. Then $T$ acts irreducibly on $Z$ and on $R / Z$. By Lemma 3.8, for $V$ an irreducible $\mathbb{F}_{p} B$-module (i.e. a $T$-module),

$\operatorname{dim} H^{2}(B, V) \leq \operatorname{dim} H^{2}(B / Z, V)+\operatorname{dim} H^{2}(Z, V)^{B}+\operatorname{dim} H^{1}\left(B / Z, H^{1}(Z, V)\right)$.

Similarly, $\operatorname{dim} H^{2}(B / Z, V) \leq \operatorname{dim} H^{2}(R / Z, V)^{T}$. Since $V$ is a trivial $R$-module, $H^{2}(R / Z, V)^{T}=0$ unless $V \cong R / Z$ or $V$ is a constituent of $\wedge^{2}(R / Z)$. We argue as usual to show that $\wedge^{2}(R / Z)$ is multiplicity free (and does not surject onto $R / Z$ ). It follows that $\operatorname{dim} H^{2}(R / Z, V)^{T} \leq \operatorname{dim}_{\operatorname{End}_{T}}(V) \cong V$ (as vector spaces). The same argument shows that either $H^{2}(Z, V)^{B}=0$ or $V \cong Z$ or $V$ is a constituent of $\wedge^{2}(Z)$, and in those cases $H^{2}(Z, V)^{B} \cong V$ (as vector spaces). Finally, note that $H^{1}\left(B / Z, H^{1}(Z, V)\right) \cong H^{1}\left(B / Z, V^{*}\right)$, and so is either 0 or has dimension equal to $\operatorname{dim} V$ if $V^{*} \cong R / Z$. So we see that each term is at most $\operatorname{dim} V$, and at most two of them can be nonzero. Thus, $\operatorname{dim} H^{2}(B, V) \leq 2 \operatorname{dim} V$.

Finally, consider $G=\Re\left(3^{2 k+1}\right), k>1$. See [46, 47] for properties of $G$.

If $r=2$, then $R$ is contained in $H:=C_{2} \times \operatorname{PSL}(2, q)$. Let $V$ be a nontrivial irreducible $F H$-module. By Lemma 3.10, $H^{2}(H, V) \cong H^{2}(\operatorname{PSL}(2, q))$. Similarly, if $V$ 
is trivial, Lemma 3.10 implies that $H^{2}(H, V) \cong H^{2}\left(C_{2}, F\right) \oplus H^{2}(\operatorname{PSL}(2, q), F)$ and so is 2-dimensional. By Lemma 7.3, it follows that $\operatorname{dim} H^{2}(H, V) \leq 2 \operatorname{dim} V$. If $r>3$, then $R$ is cyclic and the result holds by Lemma 3.5. If $r=3$, then a Borel subgroup is $T R$ where $T$ is cyclic of order $q-1$. Moreover, there are normal $T$-invariant subgroups $1=R_{0}<R_{1}<R_{2}<R_{3}=R$ such that $T$ acts irreducibly on each successive quotient (acting faithfully on the first and last quotients and acting via a group of order $(q-1) / 2$ on the middle quotient). Furthermore, $R_{2}$ is elementary abelian. Let $V$ be an irreducible $B$-module. Then, by Lemma 3.8,

$$
\begin{gathered}
\operatorname{dim} H^{2}(B, V) \leq \operatorname{dim} H^{2}\left(B / R_{2}, V\right)+\operatorname{dim} H^{2}\left(R_{2}, V\right)^{B} \\
+\operatorname{dim} H^{1}\left(B / R_{2}, H^{1}\left(R_{2}, V\right)\right),
\end{gathered}
$$

and

$$
\operatorname{dim} H^{2}\left(B / R_{2}, V\right) \leq \operatorname{dim} H^{2}\left(R_{3} / R_{2}, V\right)^{B} .
$$

By Lemma 3.17 and Lemma 3.16, it follows that $\operatorname{dim} H^{2}\left(R_{3} / R_{2}, V\right)^{B} \leq \operatorname{dim} V$ and $\operatorname{dim} H^{2}\left(R_{2}, V\right)^{B} \leq \operatorname{dim} V$. Finally, consider the final term on the right. We can write $R_{2}=W(\alpha) \oplus W(\beta)$ as a direct sum of the $T$-eigenspaces with characters $\alpha$ and $\beta$ (of orders $q-1$ and $(q-1) / 2$ ). Write $V=W(\gamma)$ as a $T$-module. Then $H^{1}\left(R_{2}, V\right)$ is a direct sum of modules $W\left(\alpha^{-1} \gamma^{\prime}\right)$ and $W\left(\beta^{-1} \gamma^{\prime}\right)$ where $\gamma^{\prime}$ is a Frobenius twist of $\gamma$. Since $T$ has order coprime to the characteristic, we see that $\operatorname{dim} H^{2}\left(B / R_{2}, V\right)$ will be the multiplicity of $R_{3} / R_{2}=W(\alpha)$ in $H^{1}\left(R_{2}, V\right)$. The comments above show that this multiplicity is 0 unless $\gamma$ is a product of two twists of $\alpha$ or a twist of $\alpha$ times a twist of $\beta$. It follows that the multiplicity in these cases is 1 and $\operatorname{dim} H^{2}(B, V) \leq \operatorname{dim} V$. Thus, $\operatorname{dim} H^{2}(B, V) \leq 3 \operatorname{dim} V$ as required.

We now consider the groups of rank 2, subdividing them into two classes. The classical groups of rank 2 will arise in the consideration of higher rank groups and so we need better bounds. The remaining cases do not occur as Levi subgroups in higher rank groups and so do not impact any of our gluing arguments.

Lemma 9.2. (1) If $G=\operatorname{SL}(3, q)$, then $h(G) \leq 3 / 2$.

(2) If $G=\mathrm{SU}(4, q)$, then $h(G) \leq 9 / 4$.

(3) If $G=\mathrm{SU}(5, q)$, then $h(G) \leq 4$.

(4) If $G=\mathrm{Sp}_{4}(q)$, then $h(G) \leq 3$.

Proof. We handle the various groups separately proving somewhat better results. The result for $\mathrm{SL}(3, q)$ is a special case of Theorem 7.4. Let $\mathbb{F}_{q}$ be the field of definition of the group with $q=p^{e}$. Let $r$ be a prime, $R$ be a Sylow $r$-subgroup of $G$ and $F=\mathbb{F}_{r}$. If $M$ is a trivial $F G$-module, the result is clear (because we know the Schur multiplier [16, pp. 312-313]). So it suffices to consider nontrivial irreducible $\mathbb{F} G$-modules. 
Case 1. $G=\mathrm{SU}(4, q)$.

If $r \neq p$ does not divide $q$, then the argument is identical to that given for $G=\operatorname{SL}(4, q)$. Suppose that $r=p$. Let $P$ be the stabilizer of a totally singular 2-space. So $P=L Q$ where $L=\operatorname{GL}\left(2, q^{2}\right)$ and $Q$ is an irreducible $\mathbb{F}_{q} L$-module of order $q^{4}$. By Lemma 3.8,

$\operatorname{dim} H^{2}(G, M) \leq \operatorname{dim} H^{2}\left(L, M^{Q}\right)+\operatorname{dim} H^{2}(Q, M)^{L}+\operatorname{dim} H^{1}\left(L, H^{1}(Q, M)\right)$.

If $q=2,3$, we apply [42]. So assume that $q>3$. Let $Z=Z(L)$. By Corollary 3.12, $H^{1}\left(L, H^{1}(Q, M)\right)=H^{1}\left(L, H^{1}(Q, M)^{Z}\right)=H^{1}\left(L, \operatorname{Hom}_{Z}(Q, M)\right)$. Since $\operatorname{dim} H^{1}(\operatorname{SL}(2, q), W) \leq(\operatorname{dim} W) / 2$ by $(1.2), \operatorname{dim} H^{1}\left(L, H^{1}(Q, M)\right) \leq$ $\operatorname{dim} M$. By Theorems 7.2 and 7.3, $\operatorname{dim} H^{2}(\operatorname{SL}(2, q), W) \leq(\operatorname{dim} W) / 2$ and so $\operatorname{dim} H^{2}\left(L, M^{Q}\right) \leq\left(\operatorname{dim} M^{Q}\right) / 2 \leq(\operatorname{dim} M) / 4$. Since $\wedge^{2}(Q)$ is multiplicity free (arguing exactly as in Lemma 3.17), the middle term is certainly at most $\operatorname{dim} M$ and so $\operatorname{dim} H^{2}(G, M) \leq(9 / 4)(\operatorname{dim} M)$.

Case 2. $G=\mathrm{SU}(5, q)$.

First consider the case $r \neq p$ and $r>2$. If $r$ does not divide $q+1$, then either $R$ is cyclic or $R$ embeds in $\mathrm{SU}(3, q)$ or $\mathrm{SU}(4, q)$ and the result follows.

Suppose that $r \mid(q+1)$. Then $R$ is contained in $H$, the stabilizer of an orthonormal basis. In particular, $H$ has a normal abelian subgroup $N$ that is homogeneous of rank 4 with $H / N=S_{5}$. Let $V$ be an irreducible $F H$-module. By Lemma 3.8,

$\operatorname{dim} H^{2}(H, V) \leq \operatorname{dim} H^{2}\left(S_{5}, V^{N}\right)+\operatorname{dim} H^{2}(N, V)^{S_{5}}+\operatorname{dim} H^{1}\left(S_{5}, H^{1}(N, V)\right)$.

If $N$ acts nontrivially on $V$, then Lemma 3.12 implies that $H^{2}(H, V)=0$. So assume that this is not the case.

Since $S_{5}$ has a cyclic Sylow $r$-subgroup, the first term on the right is at most 1 by Lemma 3.5. Since $N$ does not have a 1-dimensional quotient (as an $S_{5}$-module), it follows that $\operatorname{dim} H^{2}\left(H, \mathbb{F}_{r}\right) \leq 1$. So we may assume that $\operatorname{dim} V>1$, and so $\operatorname{dim} V \geq 3$.

Recall that $\operatorname{dim} H^{2}(N, V)^{S_{5}} \leq \operatorname{dim} \operatorname{Hom}_{S_{5}}(N, V)+\operatorname{dim} \operatorname{Hom}_{S_{5}}\left(\wedge^{2} N, V\right)$. So if $V$ is a not a quotient of either $N$ or $\wedge^{2}(N)$, then $\operatorname{dim} H^{2}(H, V) \leq 1 \leq(1 / 3) \operatorname{dim} V$. So assume that $V$ is a quotient of either $N$ or $\wedge^{2}(N)$.

If $r=5$, the only quotients of $N$ and $\wedge^{2}(N)$ are 3-dimensional. Since $\operatorname{dim} N=4$ and $\operatorname{dim} \wedge^{2}(N)=6$, it follows that $\operatorname{dim} H^{2}(H, V) \leq 4=(4 / 3) \operatorname{dim} V$.

So assume that $r \neq 5$. If $V$ is a quotient of $N$, then $V$ is the irreducible summand of the permutation module for $S_{5}$. Thus, $H^{2}\left(S_{5}, V\right)=0$ by Lemma 3.4. By dimension, it is clear that $\operatorname{dim} \operatorname{Hom}_{S_{5}}(N, V)+\operatorname{dim}_{H_{0}} S_{S_{5}}\left(\wedge^{2} N, V\right) \leq 2$, whence the result.

If $V$ is a nontrivial quotient of $\wedge^{2}(N)$ and is not a quotient of $N$, then the same argument shows that $\operatorname{dim} H^{2}(H, V) \leq 3$.

$H^{2}\left(S_{5}, V\right)=0$ if $V$ is 1-dimensional and $\operatorname{dim} V \geq 3$ otherwise, this implies that $\operatorname{dim} H^{2}\left(S_{5}, V^{N}\right) \leq(\operatorname{dim} V) / 3$. This same argument shows that $\operatorname{dim} H^{1}\left(S_{5}, W\right) \leq$ 
$(\operatorname{dim} W) / 3$ for any $F S_{5}$-module and so $H^{1}\left(S_{5}, H^{1}(N, V)\right) \leq(\operatorname{dim} N)(\operatorname{dim} V) / 3 \leq$ $(4 / 3)(\operatorname{dim} V)$.

Consider the case that $r=2 \neq p$. Then $R \leq H:=\mathrm{GU}(4, q)$. We use the result for $N:=\mathrm{SU}(4, q)$ and Lemma 3.8. So

$$
\begin{gathered}
\operatorname{dim} H^{2}(G, M) \leq \operatorname{dim} H^{2}\left(H / N, M^{N}\right)+\operatorname{dim} H^{2}(N, M)^{H} \\
+\operatorname{dim} H^{1}\left(H / N, H^{1}(N, M)\right) .
\end{gathered}
$$

This gives $\operatorname{dim} H^{2}(G, M) \leq 4 \operatorname{dim} M$ as above.

Finally, consider the case that $r=p$. Let $P$ be the stabilizer of a totally singular 2-space. Then $P=L U$ where $L$ is the Levi subgroup of $P$ and $U$ is the unipotent radical. Let $J=\operatorname{SL}\left(2, q^{2}\right) \leq L \cong \operatorname{GL}\left(2, q^{2}\right)$ and $Z=Z(L)$ cyclic of order $q^{2}-1$. Also, note that $W=[U, U]$ is irreducible of order $q^{4}$ and $X:=U / W$ is an irreducible 2-dimensional module (over $F_{q^{2}}$ ) and that $W$ is an irreducible 4-dimensional module over $\mathbb{F}_{q}$ - it is isomorphic to $X \otimes X^{(q)}$ (which is defined over $\mathbb{F}_{q}$ ).

Let $V$ be an irreducible $F P$-module. By Lemma 3.8,

$$
\begin{gathered}
\operatorname{dim} H^{2}(P, V) \leq \operatorname{dim} H^{2}(P / W, V)+\operatorname{dim} H^{2}(W, V)^{P} \\
+\operatorname{dim} H^{1}\left(P / W, H^{1}(W, V)\right) .
\end{gathered}
$$

Consider the first term on the right hand side of the inequality. By Lemma 3.8,

$$
\begin{gathered}
\operatorname{dim} H^{2}(P / W, V) \leq \operatorname{dim} H^{2}(L, V)+\operatorname{dim} H^{2}(U / W, V)^{L} \\
+\operatorname{dim} H^{1}\left(L, H^{1}(U / W, V)\right) .
\end{gathered}
$$

Note that $P / W$ is very similar to a maximal parabolic subgroup of $\operatorname{SL}\left(3, q^{2}\right)$. Arguing precisely as in that case, we see that $\operatorname{dim} H^{2}(P / W, V) \leq \operatorname{dim} V$.

Now consider the middle term. It is straightforward to see (arguing as in the proof of Lemma 3.17) that $\wedge^{2}(W)$ is multiplicity free and has no composition factors isomorphic to $W$, whence the middle term has dimension at most $\operatorname{dim} V$.

Finally, consider the last term on the right. Set $Y=H^{1}(W, V) \cong W^{*} \otimes V$. By Lemma3.13, $\operatorname{dim} H^{1}(P / W, Y) \leq \operatorname{dim} H^{1}(L, Y)+\operatorname{dim} \operatorname{Hom}_{L}(U / W, Y)$. By Corollary 3.12, $H^{1}(L, Y) \cong H^{1}\left(L, Y^{Z}\right)$. Note that $\operatorname{dim} Y^{Z} \leq 2 \operatorname{dim} V$ and so by (1.2), it follows that $\operatorname{dim} H^{1}(L, Y) \leq \operatorname{dim} H^{1}\left(J, Y^{Z}\right) \operatorname{dim} V$. Thus, $\operatorname{Hom}_{L}(U / W, Y) \cong$ $\operatorname{Hom}_{L}(U / W \otimes W, V)$.

Let $\lambda$ be the fundamental dominant weight for $J$. So $U / W=X=X(\lambda)$ (the natural module over $\mathbb{F}_{q^{2}}$ ). Note that $U$ is an $\mathbb{F}_{q} J$-module satisfying $U \otimes_{\mathbb{F}_{q}} \otimes F_{q^{2}} \cong$ $X \otimes_{\mathbb{F}_{q^{2}}} X^{(q)}$. It is straightforward to see that $U / W \otimes W$ modulo its radical is multiplicity free. Thus $\operatorname{Hom}_{L}(U / W, Y)$ is either 0 or is isomorphic to $\operatorname{End}_{L}(V)$, and so has dimension at most $\operatorname{dim} V$. It follows that $\operatorname{dim} H^{2}(P, V) \leq 4 \operatorname{dim} V$. 
Case 3. $G=\operatorname{Sp}(4, q)$.

If $r>3$ and $r \neq p$, then $R$ is abelian of rank 2, whence $\operatorname{dim} H^{2}(G, M) \leq$ $3 \operatorname{dim} M$ by Lemma 3.16 .

If $3 \geq r \neq p$, then $R$ is contained in $J$, the stabilizer of a pair of orthogonal nondegenerate 2 -spaces. If $r=3$, this implies that

$$
\operatorname{dim} H^{2}(G, M) \leq \operatorname{dim} H^{2}(\operatorname{SL}(2, q) \times \operatorname{SL}(2, q), M) \leq \operatorname{dim} M
$$

by $\S 7$ and Lemma 3.10. If $r=2$, then this shows that $\operatorname{dim} H^{2}\left(J^{\prime}, M\right) \leq \operatorname{dim} M$. By Lemma 3.8,

$$
\begin{gathered}
\operatorname{dim} H^{2}(J, M) \leq \operatorname{dim} H^{2}\left(J / J^{\prime}, M^{J^{\prime}}\right)+\operatorname{dim} H^{2}\left(J^{\prime}, M\right)^{J} \\
+\operatorname{dim} H^{1}\left(J / J^{\prime}, H^{1}\left(J^{\prime}, M\right)\right),
\end{gathered}
$$

and so $\operatorname{dim} H^{2}(J, M)<3 \operatorname{dim} M$.

If $p=r$, then $R \leq P$, the stabilizer of a totally singular 2-space. Write $P=L Q$ where $L$ is a Levi subgroup and $Q$ the unipotent radical. Note $Q$ is elementary abelian of order $q^{3}$. By Lemma 3.8,

$\operatorname{dim} H^{2}(P, M) \leq \operatorname{dim} H^{2}\left(L, M^{Q}\right)+\operatorname{dim} H^{2}(Q, M)^{L}+\operatorname{dim} H^{1}\left(L, H^{1}(Q, M)\right)$.

The first term on the right is at most $\operatorname{dim} M^{Q}$ (by the result for $\operatorname{SL}(2, q)$ ) and is at most $(\operatorname{dim} M) / 2$. Arguing as for $\operatorname{SL}(3, q), \operatorname{dim} H^{1}\left(L, H^{1}(Q, M)\right) \leq(3 / 2) \operatorname{dim} M$. By Lemma 3.16, the middle term is at most $\operatorname{dim} M$, whence $\operatorname{dim} H^{2}(G, M)<3 \operatorname{dim} M$.

We now consider the remaining rank 2 groups.

Lemma 9.3. Let $G$ be a quasisimple finite group of Lie type and rank 2. Then $\hat{r}(G) \leq 6$.

Proof. By the preceding lemma, we may assume that $G$ is one of $G_{2}(q),{ }^{3} D_{4}(q)$ or ${ }^{2} F_{4}(q)^{\prime}$. Let $p$ be the prime dividing $q$. Note that $p=2$ in the last case.

Since $G_{2}(2) \cong \operatorname{PSU}(3,3)$, we assume that $q>2$ if $G=G_{2}(q)$. We also note that a presentation is known for ${ }^{2} F_{4}(2)^{\prime}$ which gives the result (cf. [51]), so we also assume that $q>2$ in that case.

Let $r$ be a prime, $F$ a field of characteristic $r$ and $M$ an irreducible $F G$-module. Let $R$ be a Sylow $r$-subgroup of $G$.

Case $1 . G=G_{2}(q), q>2$.

If $r \neq p$ and $r>3$, then $R$ is contained in a maximal torus (since the order of $R$ is prime to the order of the Weyl group) and so $R$ is abelian of rank at most 2, whence $\operatorname{dim} H^{2}(G, M) \leq 3 \operatorname{dim} M$ by Lemma 3.16. If $p \neq r \leq 3$, then $R$ is contained in $L$ with $L \cong \operatorname{SL}(3, q) .2$ or $\mathrm{SU}(3, q) .2$ (for example, noting that the only prime 
dividing the indices of both of these subgroups is $p$ ). If $r \neq 2$, the result follows from the corresponding result for $L$. If $r=2$, by Lemma $3.8 \operatorname{dim} H^{2}(L, V) \leq$ $\operatorname{dim} H^{2}\left(L / J, V^{J}\right)+\operatorname{dim} H^{2}(J, V)^{L}+\operatorname{dim} H^{1}\left(L / J, H^{1}(J, V)\right)$, where $J$ is the derived subgroup of $L$ and $V$ is an $F L$-module. If $V$ is trivial, then this gives $\operatorname{dim} H^{2}(L, V) \leq 1$. Otherwise, $V^{J}=0$, and $\operatorname{dim} H^{2}(L, V) \leq \operatorname{dim} H^{2}(J, V)+$ $\operatorname{dim} H^{1}(J, V)<4 \operatorname{dim} V$. So $\operatorname{dim} H^{2}(G, M) \leq 4 \operatorname{dim} M$.

Now assume that $r=p$. Let $R \leq P$ be a maximal parabolic subgroup. Write $P=L Q$ where $L$ is a Levi subgroup and $Q$ is the unipotent radical. We may choose $P$ so that $Q$ has a normal subgroup $Q_{1}$ with $Q / Q_{1}$ and $Q_{1}$ each elementary abelian (of dimension 2 or 3 over $\mathbb{F}_{q}$ ). Let $V$ be an irreducible $F P$-module. It suffices by Lemma 3.6 to prove the bound in the lemma for $P$.

By Lemma 3.8,

$$
\operatorname{dim} H^{2}(P, V) \leq \operatorname{dim} H^{2}(L, V)+\operatorname{dim} H^{2}(Q, V)^{L}+\operatorname{dim} H^{1}\left(L, H^{1}(Q, V)\right) .
$$

Note that $X:=H^{1}(Q, V)=\operatorname{Hom}(Q, V)=\operatorname{Hom}(W, V)$, where $W=Q / Q_{1}$ has order $q^{2}$. If $q$ is prime, then $\operatorname{dim} X \leq 2 \operatorname{dim} V$, and so by (1.2), $\operatorname{dim} H^{1}(L, X) \leq$ $\operatorname{dim} V$. If $q$ is not prime, then $Z=Z(L)$ acts nontrivially on $W$ and so $H^{1}(L, X)=$ $H^{1}\left(L, X^{Z}\right)$ by Corollary 3.12. Since $\operatorname{dim} X^{Z} \leq 2 \operatorname{dim} V$, the same bound holds in this case. By the result for $\operatorname{SL}(2, q)$ (Theorems 7.2 and 7.3), $\operatorname{dim} H^{2}(L, V) \leq$ $(1 / 2)(\operatorname{dim} V)$. So to finish this case, it suffices to show that $\operatorname{dim} H^{2}(Q, V)^{L} \leq$ $(5 / 2)(\operatorname{dim} V)$.

By Lemma 3.8,

$$
\begin{array}{r}
\operatorname{dim} H^{2}(Q, V) \leq \operatorname{dim} H^{2}\left(Q / Q_{1}, V\right)+\operatorname{dim} H^{2}\left(Q_{1}, V\right) \\
+\operatorname{dim} H^{1}\left(Q / Q_{1}, H^{1}\left(Q_{1}, V\right)\right) .
\end{array}
$$

The proof of this inequality (either using a spectral sequence or more directly in [29]) shows that we have the same inequality after taking $L$-fixed points. Using Lemma 3.16 and arguing as usual, we see that the sum of the first two terms on the right is at most $\operatorname{dim} V$. Similarly, the right-most term is $\operatorname{Hom}\left(Q / Q_{1} \otimes Q_{1}^{*}, V\right)$ and the dimension of the $L$-fixed points is at most $\operatorname{dim} V$. The result follows.

Case 2. $G={ }^{3} D_{4}(q)$.

First suppose $r \neq p$. If $p \neq r>3$, then $R$ is contained in a maximal torus and is abelian. By inspection, $R$ has rank at most 2 and so $\operatorname{dim} H^{2}(G, M) \leq 3 \operatorname{dim} M$. If $r=3$, then $R \leq H$, the central product of $\mathrm{SL}(2, q) \circ \mathrm{SL}\left(2, q^{3}\right)$, whence we can use the bounds in $\S 7$ (obtaining a bound of $4 \operatorname{dim} M$ ). If $r=2$, then $R \leq N_{G}(H)$ and $H$ has index 2 in $N_{G}(H)$. The bound for $H$ shows that $\operatorname{dim} H^{2}\left(N_{G}(H), M\right) \leq$ $5 \operatorname{dim} M$.

If $r=p$, then $R \leq P=L Q$ with $P$ a maximal parabolic, $Q$ its unipotent radical and $L$ a Levi subgroup with simple composition factor $\operatorname{SL}\left(2, q^{3}\right)$. Then 
$|Z(Q)|=q$ and $Q / Z(Q)$ is the tensor product of the three twists of the natural module for $\operatorname{SL}\left(2, q^{3}\right)\left(\right.$ over $\left.\mathbb{F}_{q}\right)$ ). We argue as in the previous case to see that $\operatorname{dim} H^{2}(G, M) \leq 5 \operatorname{dim} M$.

Case 3. $G={ }^{2} F_{4}(q), q>2^{\prime}$.

First suppose that $r>3$. Then $R$ is abelian of rank at most 2 (by inspection of the maximal tori - see [38]), and so by Lemma $3.16 \operatorname{dim} H^{2}(G, M) \leq 3 \operatorname{dim} M$.

Note that $G$ contains a subgroup $H \cong S U(3, q)$ (see [38]). If $r=3$, then $R \leq H$ and so $\operatorname{dim} H^{2}(G, M) \leq \operatorname{dim} H^{2}(\operatorname{SU}(3, q), M) \leq 3 \operatorname{dim} M$ by Lemma 9.1.

If $r=p=2$, then $R \leq P=L Q$ with $P$ a maximal parabolic, $L=S z(q) \times C_{q-1}$ its Levi subgroup and unipotent radical $Q$. There is a sequence of normal subgroups $Q_{1}<Q_{2}<Q$ with elementary abelian quotients of order $q, q^{4}$ and $q^{5}$ respectively. We argue as above and conclude that $\operatorname{dim} H^{2}(G, M) \leq 5 \operatorname{dim} M$.

We consider two families of rank three groups that are used in the bounds for $F_{4}(q)$ and ${ }^{2} E_{6}(q)$.

Lemma 9.4. If $G=\mathrm{Sp}(6, q)$ or $\mathrm{SU}(6, q)$, then $h(G) \leq 6$.

Proof. The proofs are similar to the rank 2 cases and since the bounds are quite weak, we only sketch the proof.

Let $F$ be a field of characteristic $r$. Let $R$ be a Sylow $r$-subgroup of $G$.

First consider $G=\operatorname{Sp}(6, q)$ with $q=p^{a}$.

If $p \neq r \geq 5$, then $R$ is abelian of rank at most 3 , whence $\operatorname{dim} H^{2}(R, M) \leq$ $6 \operatorname{dim} M$ by Lemma 3.16 .

If $r=3 \neq p$, then $R$ is a contained in the stabilizer of a totally singular 3-space and so $R \leq \operatorname{GL}(3, q)$ and the result follows by the result for $\operatorname{SL}(3, q)$ and the standard argument. If $r=2 \neq p$, then $R \leq \mathrm{Sp}(4, q) \times \mathrm{Sp}(2, q)$ and we argue as usual.

If $r=p$, then $R \leq P$, the stabilizer of a totally isotropic 3-space. Then $P=L U$ where $L=\operatorname{GL}(3, q)$ is the Levi subgroup and $U$ is elementary abelian of order $q^{6}$ (and irreducible for $L$ when $q$ is odd). We argue as usual.

Now suppose that $G=\mathrm{SU}(6, q)$ with $q=p^{a}$. First consider the case that $p \neq r$. If $r>3$ does not divide $q+1$, then $R$ is abelian of rank at most 3, whence the result holds. If $r=3$ does not divide $q+1$, then $R \leq \mathrm{GL}\left(3, q^{2}\right)$ and the result follows.

If $3 \leq r$ does divide $q+1$, then $R \leq S:=A . S_{6}$ where $A$ is isomorphic to $C_{q+1}^{5}$. The result now follows by using the bounds for $S_{6}$ and Lemma 3.8.

If $r=2 \neq p$, then $R$ stabilizes a nondegenerate 4-space. So we use the results for $\mathrm{SU}(2, q)$ and $\mathrm{SU}(4, q)$ and argue as usual.

If $r=p$, then $R \leq P$, the stabilizer of a totally singular 3-space. Note $P=L Q$ where $Q$ is the unipotent radical and $L$ the Levi subgroup. Note $Q$ is an irreducible $L$-module of order $q^{9}$ and $L \cong \operatorname{GL}\left(3, q^{2}\right)$. We argue as usual. 


\section{Groups of Lie type - the general case}

Here we essentially follow the argument in [21] but use profinite presentations.

Theorem 10.1. Let $G$ be a quasisimple finite group with $G / Z(G)$ a group of Lie type. Then $\hat{r}(G) \leq 18$.

Proof. Let $G$ be the simply connected group of the given type of rank $n$. By the results of the previous section, we may assume that $n \geq 3$. Consider the Dynkin diagram for $G$. Let $\Pi$ be the set of simple roots and write $\Pi=\left\{\alpha_{1}, \ldots, \alpha_{n}\right\}$.

First suppose that $G$ is a classical group. We assume the numbering of roots is such that the subsystem $\left\{\alpha_{1}, \ldots, \alpha_{n-1}\right\}$ is of type $A_{n-1}, \alpha_{n}$ is an end node root and is connected to only one simple root $\alpha_{j}$ in the Dynkin diagram (in the typical numbering for a Dynkin diagram, $j=n-1$ except for type $D$ when $j=n-2$ ).

Let $G_{1}$ be the subgroup generated by the root subgroups $U_{ \pm \alpha_{i}}, 1 \leq i<n$. Let $G_{2}$ be the rank 2 subgroup generated by the root subgroups $U_{ \pm \alpha_{n}}, U_{ \pm \alpha_{j}}$. Let $L_{2}$ be the rank 1 subgroup corresponding to the simple root $\alpha_{n}$. Let $L_{1}$ be the subgroup of $G_{1}$ generated by the root subgroups that commute with $L_{2}$. Note that $L_{1}$ is an SL unless $G$ has type $D_{n}$ in which case $L_{2}$ is of type $\operatorname{SL}(2) \times \operatorname{SL}(n-2)$. Let $L$ be the rank one subgroup generated by $U_{ \pm \alpha_{j}}$. Let $\langle X \mid R\rangle$ be a presentation for $G_{1}$ and $\langle Y \mid S\rangle$ be a presentation for $G_{2}$ with $X$ and $Y$ disjoint.

We give a presentation for a group $J$ with generators $X \cup Y$ and relations $R, S$, $\left[L_{1}, L_{2}\right]=1$ and we identify the copies of $L$ in $G_{1}$ and $G_{2}$. More precisely, take two generators for each $L_{i}$, express them as words in $X$ and $Y$ and impose the four commutation relations. Similarly, take our two generators for $L$ and take two words each in $X$ and $Y$ which map onto those generators of $L$ in $G$ and equate the corresponding words.

We claim $J \cong G$. Clearly, $J$ surjects onto $G$. Thus, the subgroup generated by $X$ in this presentation can be identified with $G_{1}$ and the subgroup generated by $Y$ can be identified with $G_{2}$. Now $J$ is generated by the simple root subgroups contained in $G_{1}$ or $G_{2}$. Any two of the these root subgroups (and their negatives) satisfy the Curtis-Steinberg-Tits relations (for either they are both in $G_{1}$ or $G_{2}$ or they commute by our relations since $\left[L_{1}, L_{2}\right]=1$ ). By Lemma $8.1 J$ is a homomorphic image of the universal finite group of Lie type of the given type, and the claim follows.

Note that the number of relations is $|R|+|S|+6$ (since 4 relations are required to ensure that $\left[L_{1}, L_{2}\right]=1$ and 2 relations to identify the copies of $L$ ) and the number of generators is $|X|+|Y|$. Using Lemma 3.15 and the fact that $G, G_{1}$ and $G_{2}$ are all 2-generated, we see that

$$
\hat{r}(G) \leq \hat{r}\left(G_{1}\right)+\hat{r}\left(G_{2}\right)+6-2 .
$$

Now $G_{1} \cong$ SL and so satisfies $\hat{r}\left(G_{1}\right) \leq 9$ by Corollary 8.3, and $G_{2}$ is either of type $B_{2}$ or $\mathrm{SU}(d, q)$ with $d=4$ or 5 . In particular, $\hat{r}\left(G_{2}\right) \leq 5$ by Lemma 9.2 
and (1.4). This gives $\hat{r}(G) \leq 18$ as required, and also $\hat{r}(G / Z) \leq 18$ for any central subgroup $Z$ of $G$ by Corollary 4.2 .

We now consider the exceptional groups. The idea is essentially the same, but we have to modify the construction slightly. If $G=E_{n}(q)$ with $6 \leq n \leq 8, G_{1}$ still has type $A_{n-1}, G_{2}$ has type $A_{2}$, but $L_{1}=A_{2} \times A_{n-4}$, and $L_{1}$ is generated by 2 elements, there is no difference in the analysis of the presentation. Thus, $\hat{r}(G) \leq 18$.

If $G=F_{4}$, we take $G_{1}=C_{3}$ and $G_{2}=A_{2}$. Then $L_{1}=A_{1}$. Similarly, if $G={ }^{2} E_{6}(q)$, then $G_{1}=\operatorname{SU}(6, q)$ and $G_{2}$ is of type $A_{2}$. By Lemma 9.4, $\hat{r}\left(G_{1}\right) \leq 7$. Since $\hat{r}\left(G_{2}\right) \leq 3$, we see that $\hat{r}(G) \leq 3+7+2+4-2=14$.

Now let $G$ be a quasisimple group with $G / Z(G)$ a simple finite group of Lie type. If $G$ is a homomorphic image the universal Chevalley group, then we have shown that in all cases $\hat{r}(G) \leq 18$. We need to consider the possibility that $G$ has a Schur multiplier whose order divides the characteristic of $G$. If $G / Z(G)$ is isomorphic to an alternating group, we have already proved the result. By [16, p. 313] the only groups $G / Z(G)$ that remain to be considered are $\operatorname{PSL}(3,2), \operatorname{PSL}(3,4), \operatorname{PSU}(4,2)$, $\operatorname{PSU}(6,2), \operatorname{Sp}(6,2), S z(8), \mathrm{P} \Omega^{+}(8,2), G_{2}(4), F_{4}(2)$ and ${ }^{2} E_{6}(2)$. In all these cases, we have shown that $\hat{r}(G / Z) \leq 14$ Thus, $\hat{r}(G) \leq 15$ by Corollary 4.2 .

\section{Sporadic groups}

Now let $G$ be a quasisimple sporadic group and $M$ an irreducible $\mathbb{F}_{p} G$-module. In this section, we prove:

Theorem 11.1. Let $G$ be a finite quasisimple group with $G / Z(G)$ a sporadic simple group. Then $G$ has a profinite presentation with 2 generators and 18 relations, and $\operatorname{dim} H^{2}(G, M) \leq(17.5) \operatorname{dim} M$ for any $F G$-module $M$.

One can certainly prove better bounds. We use the main result of Holt [30] to see that $\operatorname{dim} H^{2}(G / Z, M) \leq 2 e_{p}(G / Z) \operatorname{dim} M$, where $p^{e_{p}(G)}$ is the order of a Sylow $p$-subgroup of $G$. Also, for many of the groups, there is a presentation with less than 18 relations (see [51]), whence the results follow (note that in all cases the Schur multiplier is cyclic [16, p. 313]).

So we only need to deal with those sporadic groups (and their covering groups) where neither of these arguments suffices. The only cases to consider are $p=2$ and a few cases for $p=3$.

In these cases, it is more convenient to work with the simple group rather than the covering group.

The table below lists the cases that are not covered by Holt's result or by the presentations given in [51]. We give the structure of a subgroup $H$ of the simple group $S:=G / Z$ that contains a Sylow $p$-subgroup of $S$ in order to apply Lemma 3.6. 


\begin{tabular}{lccc}
$G / Z$ & $|Z|_{p}$ & $p$ & $H$ \\
\hline $\mathrm{Co}_{3}$ & 1 & 2 & $2^{4} \cdot A_{8}$ \\
$\mathrm{Co} o_{2}$ & 1 & 2 & $2^{1+8} \cdot S p(6,2)$ \\
$\mathrm{Co} o_{1}$ & 2 & 2 & $2^{11} \cdot M_{24}$ \\
$\mathrm{He}$ & 1 & 2 & $2^{6} \cdot 3 \cdot S_{6}$ \\
$\mathrm{Fi}_{22}$ & 2 & 2 & $2^{10} \cdot M_{22}$ \\
$\mathrm{Fi}_{23}$ & 1 & 2 & $2 \cdot F i_{22}$ \\
$\mathrm{Fi}_{24}^{\prime}$ & 1 & 2 & $2^{11} \cdot M_{24}$ \\
$\mathrm{Suz}_{2}$ & 2 & 2 & $2^{1+6} \cdot U_{4}(2)$ \\
$J_{4}$ & 1 & 2 & $2^{11} \cdot M_{24}$ \\
$H N$ & 1 & 2 & $2^{1+8} \cdot\left(A_{5} \times A_{5}\right) \cdot 2$ \\
$T h$ & 1 & 2 & $2^{5} \cdot L_{5}(2)$ \\
$B$ & 2 & 2 & $2^{1+22} \cdot C o_{2}$ \\
$M$ & 1 & 2 & $2^{1+24} \cdot C o_{1}$ \\
\hline$F i_{23}$ & 1 & 3 & $O^{+}(8,3) \cdot 3$ \\
$F i_{24}^{\prime}$ & 3 & 3 & $3^{1+10} \cdot U(5,2)$ \\
$B$ & 1 & 3 & $3^{1+8} \cdot 2^{1+6} \cdot U(4,2)$ \\
$M$ & 1 & 3 & $3^{8} \cdot O^{-}(8,3)$ \\
\hline
\end{tabular}

Let $G=C o_{1}$ and let $N=O_{p}(H)$.

Note that a Sylow 2-subgroup of $M_{24}$ is contained in a subgroup isomorphic to $2^{4} A_{8}$. Using the results for $A_{8}$ and the computations in [42], we see that $\operatorname{dim} H^{2}\left(M_{24}, M\right) \leq \operatorname{dim} M$. The standard arguments now yield $\operatorname{dim} H^{2}(H, M) \leq$ $3 \operatorname{dim} M$ for $M$ an $\mathbb{F}_{2} H$-module where $H=2^{11} M_{24}$, and therefore we obtain the same bound for $G$. Similar computations using the subgroups in the table show that the results hold in all the remaining cases.

By (1.1), this completes the proof of Theorem 11.1.

\section{Higher cohomology}

We have seen that $\operatorname{dim} H^{k}(G, M) \leq C \operatorname{dim} M$ for $M$ a faithful irreducible $F G$ module and $k \leq 2$. In fact, it is unknown whether there is an absolute bound $C_{k}$ for $\operatorname{dim} H^{k}(G, M)$ for $M$ an absolutely irreducible $F G$-module with $G$ simple. It was conjectured by the first author over twenty years ago that this was the case for $k=1$. Indeed, there are no examples known with $\operatorname{dim} H^{1}(G, M)>3$ for $M$ an absolutely irreducible $F G$-module and $G$ a finite simple group. So we ask again: 
Question 12.1. For which $k$ is it true that there is an absolute constant $C_{k}$ such that $\operatorname{dim} H^{k}(G, V)<C_{k}$ for all absolutely irreducible $F G$-modules $V$ and all finite simple groups $G$ with $F$ an algebraically closed field (of any characteristic)?

See [12] for some recent evidence related to this conjecture.

A slightly weaker version of this question for $k=1$ is relevant to an old conjecture of Wall. His conjecture is that the number of maximal subgroups of a finite group $G$ is less than $|G|$. If we consider groups of the form $V H$ with $V$ an irreducible $\mathbb{F}_{p} H$-module, a special case of Wall's conjecture (and likely the hardest case is):

Question 12.2. If $V$ is an irreducible $\mathbb{F}_{p} G$-module with $G$ finite, is $\left|H^{1}(G, V)\right|<$ $|G|$ ?

This is true for $G$ solvable [48], and in that case is essentially equivalent to Wall's conjecture.

We now give some examples to show that the analog of Theorem $\mathrm{C}$ does not hold for $H^{k}, k>2$.

Let $F$ be an algebraically closed field of characteristic $p>0$.

Let $S$ be a nonabelian finite simple group such that $p$ divides both the order of $S$ and the order of its outer automorphism group. Let $L$ be a subgroup of $\operatorname{Aut}(S)$ containing $S$ with $L / S$ of order $p$. Let $W$ be an irreducible $F S$-module with $H^{1}(S, W) \neq 0$. Note that if $x \in S$ has order $p$, then all Jordan blocks of $x$ have size $p$ in any projective $F S$-module. In particular, the trivial module is not projective and so $H^{1}(S, W) \neq 0$ for some irreducible module $W$. Obviously such a $W$ can not be the trivial module. Let $U=W_{S}^{L}$. Then either $U$ is irreducible and $H^{1}(L, U) \cong H^{1}(S, W) \neq 0$ by Lemma 3.4, or each of the $p$ composition factors of $U$ (as an $L$-module) is isomorphic to $W$ as $F S$-modules. Since $H^{1}(L, U) \neq 0$, some irreducible $L$-composition factor of $U$ also has nontrivial $H^{1}$ by Lemma 3.3. In either case, we see that there exists an irreducible faithful $F L$-module $V$ with $H^{1}(L, V) \neq 0$ and $H^{1}(L, F) \cong F$.

Let $G=L<C_{t}$ and let $N<G$ be the direct product $L_{1} \times \cdots \times L_{t}$ with $L_{i} \cong L$. Let $X=V \otimes F \otimes \cdots \otimes F$. So $X$ is an irreducible $F N$-module.

By Lemma 3.10, for $k \geq 3$,

$$
\operatorname{dim} H^{k}(N, X) \geq \operatorname{dim} H^{1}(L, V) \cdot\left(\begin{array}{l}
t-1 \\
k-1
\end{array}\right) \geq c_{k} t^{k-1} .
$$

for some constant $c_{k}$. Thus, for $t$ sufficiently large, $\operatorname{dim} H^{k}(N, X)>d_{k} t^{k-1} \operatorname{dim} X$ for the constant $\left.d_{k}:=\left(c_{k} \operatorname{dim} H^{1}(L, V)\right) /(\operatorname{dim} V)\right)$.

Similarly, $\operatorname{dim} H^{2}(N, X)=\operatorname{dim} H^{2}(L, V)+(t-1) \operatorname{dim} H^{1}(L, V) \geq t-1$.

Now let $M=X_{N}^{G}$. By Lemma 3.4, $\operatorname{dim} H^{k}(G, M)=\operatorname{dim} H^{k}(N, X)$.

We record the following consequence for $k=2$. 
Theorem 12.3. Let $F$ be an algebraically closed field of characteristic $p>0$. There is a constant $e_{p}>0$ such that if $d$ is a positive integer, then there exist a finite group $G$ and an irreducible faithful $F G$-module with $\operatorname{dim} H^{2}(G, M) \geq e_{p} \operatorname{dim} M$ and $\operatorname{dim} M>d$.

In particular, we see that $\operatorname{dim} H^{2}(G, M)$ can be arbitrarily large for $M$ an irreducible faithful $F G$-module in any characteristic. Another way of stating the previous result is that for a fixed $p$,

$$
u(p):=\limsup _{\operatorname{dim} M \rightarrow \infty} \frac{\operatorname{dim} H^{2}(G, M)}{\operatorname{dim} M}>0 .
$$

Here we are allowing any finite group $G$ with $M$ any irreducible faithful $\mathbb{F}_{p} G$-module. The scarce evidence suggests:

Conjecture 12.4. $\lim _{p \rightarrow \infty} u(p)=0$.

If $k>2$, we obtain:

Lemma 12.5. Keep notation as above.

(1) $M$ is an irreducible faithful $\mathbb{F}_{p} G$-module with $\operatorname{dim} M=t \operatorname{dim} X$.

(2) $\operatorname{dim} H^{k}(G, M) \geq d_{k} t^{k-2} \operatorname{dim} M$.

(3) There exists a constant $e_{k}>0$ such that $\operatorname{dim} H^{k}(G, M) \geq e_{k}(\operatorname{dim} M)^{k-1}$.

Proof. Note that $M$ is a direct sum of $t$ nonisomorphic irreducible $F N$-modules that are permuted by $G$ and so $M$ is irreducible. Since $N$ is the unique minimal normal subgroup of $G$ and does not act trivially on $M, G$ acts faithfully on $M$. Now (2) follows by the discussion above and by Lemma 3.4. Similarly, (3) follows with $e_{k}=c_{k} /(\operatorname{dim} V)^{k-1}$.

So we have shown:

Theorem 12.6. Let $k$ be a positive integer. If $k \geq 3$, there exist finite groups $G$ and faithful absolutely irreducible $F G$-modules $M$ with $\operatorname{dim} H^{k}(G, M) /(\operatorname{dim} M)^{k-2}$ arbitrarily large.

Our reduction methods in Section 5.3 give very weak bounds for the dimension of $H^{k}(G, M)$ with $M$ faithful and irreducible in terms of the bounds for the simple groups. We ask whether our examples are the best possible:

Question 12.7. For which positive integers $k$ is it true that there is an absolute constant $d_{k}$ such that $\operatorname{dim} H^{k}(G, V)<d_{k}(\operatorname{dim} V)^{k-1}$ for all absolutely irreducible faithful $F G$-modules $V$ and all finite groups $G$ with $F$ an algebraically closed field (of any characteristic)? 
For $k=1$, the question reduces to the case of simple groups. Theorem $\mathrm{C}$ says that we can take $d_{2}=18.5$.

\section{Profinite versus discrete presentations}

In this section, we consider discrete and profinite presentations for finite groups. Recall that $r(G)$ (respectively $\hat{r}(G)$ ) denotes the minimal number of relations required in a presentation (respectively profinite presentation) of a finite group $G$. In fact, if $G=F / N$ is a discrete presentation of $G$ (i.e. $F$ is a free group), then $G=\hat{F} / \bar{N}$, where $\hat{F}$ is the profinite completion of $F$ and $\bar{N}$ is the closure of $N$ in $\hat{F}$. So $\hat{F} / \bar{N}$ is a profinite presentation for $G$. Indeed, every profinite presentation of $G$ can be obtained this way.

Let $R=N /[N, N]$ and for a prime $p$, set $R(p)=N /[N, N] N^{p}$. So $R$ (resp. $R(p)$ ) is the relation (resp. $p$-relation) module of $G$ with respect to the given presentation. Denote by $d_{F}(N)$ the minimal number of generators required for $N$ as a normal subgroup of $F$ and $d_{G}(R)$ (resp. $d_{G}(R(p))$ the minimal number of generators required for $R$ (resp. $R(p)$ ) as a $\mathbb{Z} G$-module. Similarly, define $\hat{d}_{\hat{F}}(\bar{N})$ to be the minimal number of generators required for $\bar{N}$ as a closed normal subgroup of $\hat{F}$.

A theorem of Swan [17, Theorem 7.8] asserts that $d_{G}(R)=\max _{p} d_{G}(R(p))$, and Lubotzky [33] showed that $\hat{d}_{\hat{F}}(\bar{N})=\max _{p} d_{G}(R(p))$. So altogether $\hat{d}_{\hat{F}}(\bar{N})=$ $d_{G}(R)$. Moreover, it is shown in [33] that $\hat{r}(G)=\hat{d}_{\hat{F}}(\bar{N})$ for any minimal presentation of $G$, i.e. a presentation in which $d(F)=d(G)$ (see also Lemma 3.15). The analogous property for discrete presentations of finite groups is not known and fails for infinite groups (cf. [17, p. 2]).

The long standing open problem whether $d_{F}(N)=d_{G}(R)$ (see [17, p. 4]) therefore has an equivalent formulation:

Question 13.1. Is $d_{F}(N)=\hat{d}_{\hat{F}}(\bar{N})$ ?

A variant of this question is even more interesting:

Question 13.2. Is $\hat{r}(G)=r(G)$ ?

Of course, a positive answer to Question 13.1 would imply a positive answer to Question 13.2, but not conversely.

A weaker version of Question 13.1 is:

Question 13.3. Given a presentation $G=F / N=\hat{F} / \bar{N}$ of the finite group $G$, are there $d_{\hat{F}}(\bar{N})=d_{G}(R)$ elements of $N$ which generate $\bar{N}$ as a closed normal subgroup of $\hat{F}$ ? 
In light of the above discussion, it is not surprising that our results in [21] and in the current paper give better estimates for profinite presentations of finite simple groups than for discrete presentations. Theorem A ensures that all finite simple groups have profinite presentations with at most 18 relations. We investigate discrete presentations in [21] and [22]. In [22], we worry less about the total length of relations and prove:

Theorem 13.4. Every finite simple group, with possible exception of ${ }^{2} G_{2}\left(3^{2 k+1}\right)$, has a presentation with 2 generators and at most 100 relations.

Of course, both 18 and 100 are not optimal and indeed, as we have already observed, for many groups we know much better bounds. One may hope that 4 is the right upper bound for both types of presentations. Indeed, there is no known obstruction to the full covering group of a finite simple group having a presentation with 2 generators and 2 relations (see [50]).

Let us now turn our attention to presentations (and cohomology) of general finite groups.

If $G=F / N$ is simple (and not ${ }^{2} G_{2}(q)$ ) with $F$ free and $d(F)=2$, then by the results of [21], $N$ can be generated, as a normal subgroup of $F$, by $C$ words for some absolute constant $C$ (and the total length of the words used can be bounded in terms of $|G|$ ). Mann [39] showed that if every finite simple group can be presented with $O(\log |G|)$ relations, then every finite group could be presented with $O(d(G) \log |G|) \leq O\left((\log |G|)^{2}\right)$ relations. Of course, in [21], we proved that simple groups (with possible exception of ${ }^{2} G_{2}(q)$ ) can be presented with a bounded number of relations - but the better bound for simple groups does not translate to a better bound for all groups. Mann's argument is valid also in the profinite case and since there are no exceptions, we have:

Theorem 13.5. Let $G$ be a finite group.

(1) If $G$ has no composition factors isomorphic to ${ }^{2} G_{2}\left(3^{2 k+1}\right)$, then $G$ has a presentation with $O(d(G) \log |G|)$ relations.

(2) $G$ has a profinite presentation with $O(d(G) \log |G|)$ relations.

The example of an elementary abelian 2-group shows that one can do no better in general. Results like the above have been used to count groups of a given order (or perfect groups of a given order) and also for getting results on subgroup growth. Fortunately the profinite result is sufficient for these types of results and so the Ree groups do not cause problems. See [33].

Using the reduction of [5, Theorem 1.4] to simple groups for lengths of presentations, one sees that:

Theorem 13.6. Let $G$ be any finite group with no composition factors isomorphic to ${ }^{2} G_{2}(q)$. Then $G$ has a presentation of length $O\left((\log |G|)^{3}\right)$. 
This is essentially in [5] aside from excluding $\mathrm{SU}(3, q)$ and Suzuki groups (at the time of that paper it was not known that those groups had presentations with $\log |G|$ relations). As pointed out in [5], the constant 3 in the previous theorem cannot be improved (by considering 2-groups).

We now give some refinements of these results in the profinite setting. We first prove some results about $H^{2}$.

We need to introduce some notation. Recall that a chief factor $X$ of a finite group is a nontrivial section $A / B$ of $G$ where $B$ and $A$ are both normal in $G$ and there is no normal subgroup of $G$ properly between $A$ and $B$. Clearly $X$ is characteristically simple and so $X$ is either an elementary abelian $r$-group for some prime $r$ or $X$ is isomorphic to a direct product of copies of a nonabelian simple group and $G$ permutes these factors transitively. There is an obvious definition of isomorphism of chief factors. An appropriate version of the Jordan-Hölder theorem implies that the multi-set of chief factors coming from a maximal chain of normal subgroups of $G$ is independent of the chain.

If $X$ is a nonabelian chief factor, let $s_{p}(X)$ denote the $p$-rank of the Schur multiplier of a simple direct factor of $X$. So $s_{p}(X) \leq 2$ (and for $\left.p>3, s_{p}(X) \leq 1\right)[16$, pp. 312-313]. Let $s_{p}(G)$ denote the sum of the $s_{p}(X)$ as $X$ ranges over the nonabelian chief factors of $G$ (counting multiplicity). If $X$ is a chief factor of $G$ and is an elementary abelian $p$-group, let $\ell_{p}(X)=\log _{p}|X|$. Let $\ell_{p}(G)$ denote the sum of $\ell_{p}(X)$ as $X$ ranges over the chief factors of $G$ that are $p$-groups.

Define

$$
h_{p, 1}(G)=\max \left\{1+\operatorname{dim} H^{1}(G, V) / \operatorname{dim} V\right\},
$$

where $V$ is an irreducible $\mathbb{F}_{p} G$-module. Note that this is always bounded by $d(G)+1$ (or $d(G)$ if $V$ is nontrivial) since a derivation is determined by its images on a set of generators. We can now prove:

Theorem 13.7. Let $G$ be a finite group and $V$ an $\mathbb{F}_{p} G$-module. Then

$$
\operatorname{dim} H^{2}(G, V) \leq\left(C+s_{p}(G)+h_{p, 1}(G) \ell_{p}(G)\right) \operatorname{dim} V,
$$

where $C=18.5$ is the constant given in Theorem $C$.

Proof. Let $N$ be a minimal normal subgroup of $G$. We first claim that $h_{p, 1}(G / N) \leq$ $h_{p, 1}(G)$. Let $W$ be an irreducible $\mathbb{F}_{p}(G / N)$-module which we may consider as an $F G$-module. Let $H=W . G$. Then $N$ is normal in $H$. If $X$ is a complement to $W$ in $H / N$, then $Y$ is a complement to $W$ in $H$, where $Y$ is the preimage of $X$ in $H$. Thus, the number of complements of $W$ in $H$ is at least the number of complements of $W$ in $H / N$. So $\operatorname{dim} H^{1}(G / N, W) \leq \operatorname{dim} H^{1}(G, W)$, whence the claim.

It suffices to prove the theorem for $V$ irreducible. If $G$ acts faithfully on $V$, this follows from Theorem $C$. So we may assume that there is a minimal normal subgroup $N$ of $G$ that acts trivially on $V$. 
By Lemma 3.8,

$\operatorname{dim} H^{2}(G, V) \leq \operatorname{dim} H^{2}(G / N, V)+\operatorname{dim} H^{2}(N, V)^{G}+\operatorname{dim} H^{1}\left(G / N, H^{1}(N, V)\right)$.

Suppose that $N$ is nonabelian. Then $N$ is perfect, and so $H^{1}(N, V)=0$ by Lemma 3.9. By Lemma 3.10, $H^{2}(N, V)=\oplus H^{2}\left(L_{i}, V\right)$, where $N$ is the direct product of the $L_{i}$. Since $G$ permutes the $L_{i}$ transitively, it also permutes the $H^{2}\left(L_{i}, V\right)$, and so $H^{2}(N, V)^{G}$ embeds in $H^{2}(L, V)$ where $L \cong L_{i}$. Since $V$ is a trivial module, $\operatorname{dim} H^{2}(L, V)=\operatorname{dim} H^{2}(L, F) \operatorname{dim} V=s_{p}(N) \operatorname{dim} V$. So in this case, we have: $\operatorname{dim} H^{2}(G, V) \leq \operatorname{dim} H^{2}(G / N, V)+s_{p}(N) \operatorname{dim} V$ and the result follows by induction.

Suppose that $N$ is abelian. If $N$ is a $p^{\prime}$-group, then the last two terms in the inequality above are 0 and the result follows. So assume that $N$ is an elementary abelian $p$-group. Set $e=\ell_{p}(N)$. By definition we have $\operatorname{dim} H^{1}\left(G / N, H^{1}(N, V)\right) \leq$ $\left(h_{p, 1}(G)-1\right) e \operatorname{dim} V$. By induction, it suffices to show that $\operatorname{dim} H^{2}(N, V)^{G} \leq$ $e \operatorname{dim} V$. By Lemma 3.16,

$$
\operatorname{dim} H^{2}(N, V)^{G} \leq \operatorname{dim} \operatorname{Hom}_{G}(N, V)+\operatorname{dim} \operatorname{Hom}_{G}\left(\wedge^{2}(N), V\right) .
$$

If $V \cong N$, then clearly the number of composition factors of $\wedge^{2}(N)$ isomorphic to $V$ is at most $(e-1) / 2$, and so $\operatorname{dim} H^{2}(N, V)^{G} \leq(e+1) / 2\left(\operatorname{dim}_{\operatorname{End}_{\mathrm{G}}}(\mathrm{V})\right) \leq e \operatorname{dim} V$. If $V$ is not isomorphic to $N$, then $H^{2}(N, \bar{V})^{G}=0$, and so $\operatorname{dim} H^{2}(N, V)^{G} \leq$ $\operatorname{Hom}_{G}\left(\wedge^{2}(N), V\right)$ and by Lemma 3.18, $\operatorname{dim} H^{2}(N, V)^{G} \leq(e-1) \operatorname{dim} V$. This completes the proof.

Note that $s_{p}(G)$ is at most twice the number of nonabelian chief factors of $G$.

If we only consider $d$-generated groups, then as noted above, $h_{p, 1} \leq d+1$. Indeed, $\operatorname{dim} H^{1}(G, V) \leq(d-1) \operatorname{dim} V$ unless $V$ involves trivial modules. So one has:

Corollary 13.8. Let $G$ be a finite group with $d(G)=d$ and $V$ an $\mathbb{F}_{p} G$-module. Then $\operatorname{dim} H^{2}(G, V) \leq\left(C+s_{p}(G)+(d+1) \ell_{p}(G)\right) \operatorname{dim} V$, where $C=18.5$ is the constant given in Theorem $C$.

Now using (1.1), we can obtain results about profinite presentations. Let $h_{1}(G)$ be the maximum of $h_{p, 1}(G)$ over $p, \ell(G)$ the maximum of the $\ell_{p}(G)$ and $s(G)$ the maximum of the $s_{p}(G)$. The following is a refinement of the results mentioned in the beginning of the section.

Theorem 13.9. Let $G$ be a finite group. Then $\hat{r}(G) \leq d(G)+C+s(G)+h_{1}(G) \ell(G)$, where $C-1=18.5$ is the constant in Theorem $C$. In particular, if $d(G) \leq d$, then $\hat{r}(G) \leq d+C+s(G)+(d+1) \ell(G)$. 
This improves Theorem 13.5 in the profinite setting since $s(G)$ and $\ell(G)$ are bounded above by $\log _{2}|G|$ and $h_{1}(G) \leq d(G)+1$.

We mention some special cases that are a bit surprising.

Corollary 13.10. Let $G$ be a finite group with no abelian composition factors. Then $\hat{r}(G) \leq d(G)+19+2 s$ where $s$ is the number of chief factors of $G$.

Corollary 13.11. Let $G$ be a finite group with no abelian composition factors and no composition factors that have a nontrivial Schur multiplier. Then $\hat{r}(G) \leq d(G)+19$.

It is not clear that the previous result is true for discrete presentations and may suggest a strategy for proving that one does not always have $r(G)=\hat{r}(G)$.

\section{References}

[1] H. H. Andersen, J. Jørgensen, and P. Landrock, The projective indecomposable modules of SL $\left(2, p^{n}\right)$. Proc. London Math. Soc. (3) 46 (1983), 38-52. Zbl 0503.20013 MR 684821

[2] M. Aschbacher, Finite group theory. Corrected reprint, Cambridge Stud. Adv. Math. 10, Cambridge University Press, Cambridge 1993. Zbl 0826.20001 MR 1264416

[3] M. Aschbacher, On the maximal subgroups of the finite classical groups. Invent. Math. 76 (1984), 469-514. Zbl 0537.20023 MR 746539

[4] M. Aschbacher and R. Guralnick, Some applications of the first cohomology group. J. Algebra 90 (1984), 446-460. Zbl 0554.20017 MR 760022

[5] L. Babai, A. J. Goodman, W. M. Kantor, E. M. Luks, and P. P. Pálfy, Short presentations for finite groups. J. Algebra 194 (1997), 79-112. Zbl 0896.20025 MR 1461483

[6] A. Babakhanian, Pure Appl. Math. 11, Cohomological methods in group theory. Marcel Dekker, New York 1972. Zbl 0256.20068

[7] G. W. Bell, On the cohomology of the finite special linear groups, I, II. J. Algebra 54 (1978), 216-238, 239-259. Zbl 0389.20043 MR 511463

[8] D. J. Benson, Representations and cohomology. I. 2nd ed., Cambridge Stud. Adv. Math. 30, Cambridge University Press, Cambridge 1998. Zbl 0908.20001 MR 1644252

[9] Á. Bereczky, Maximal overgroups of Singer elements in classical groups. J. Algebra 234 (2000), 187-206. Zbl 0971.20024 MR 1799483

[10] K. S. Brown, Cohomology of groups. Grad. Texts in Math. 87, Springer-Verlag, New York 1982. Zbl 0584.20036 MR 0672956

[11] R. Burkhardt, Die Zerlegungsmatrizen der Gruppen PSL $\left(2, p^{f}\right)$. J. Algebra 40 (1976), 75-96. Zbl 0334.20008 MR 0480710

[12] E. Cline, B. Parshall, and L. Scott, Reduced standard modules and cohomology. Trans. Amer. Math. Soc., to appear. 
[13] C. W. Curtis, Central extensions of groups of Lie type. J. Reine Angew. Math. 220 (1965), 174-185. Zbl 0137.25701 MR 0188299

[14] L. Dornhoff, Group representation theory. Part B: Modular representation theory. Pure Appl. Math. 7, Marcel Dekker, New York 1972. Zbl 0236.20004 MR 0347959

[15] W. Feit, The representation theory of finite groups. North-Holland Math. Library 25, North-Holland, Amsterdam 1982. Zbl 0493.20007 MR 0661045

[16] D. Gorenstein, R. Lyons, and R. Solomon, The classification of the finite simple groups, Number 3, Almost simple $K$-groups. Math. Surveys Monogr. 40.3, Amer. Math. Soc., Providence, RI, 1998. Zbl 0890.20012 MR 1490581

[17] K. W. Gruenberg, Relation modules of finite groups. CBMS Regional Conf. Ser. in Math. 25, Amer. Math. Soc., Providence, RI, 1976. Zbl 0327.20019 MR 0457538

[18] R. M. Guralnick, Generation of simple groups. J. Algebra 103 (1986), 381-401. Zbl 0601.20013 MR 860714

[19] R. M. Guralnick and C. Hoffman, The first cohomology group and generation of simple groups. In Groups and geometries (Siena, 1996), Trends Math., Birkhäuser, Basel 1998, 81-89. Zbl 0894.20039 MR 1644977

[20] R. M. Guralnick and W. M. Kantor, Probabilistic generation of finite simple groups. J. Algebra 234 (2000), 743-792. Zbl 0973.20012 MR 1800754

[21] R. M. Guralnick, W. M. Kantor, M. Kassabov, and A. Lubotzky, Presentations of finite simple groups: a quantitative approach. J. Amer. Math. Soc. To appear.

[22] R. M. Guralnick, W. M. Kantor, M. Kassabov, and A. Lubotzky, Presentations of finite simple groups: a computational approach. Preprint.

[23] R. M. Guralnick and W. Kimmerle, On the cohomology of alternating and symmetric groups and decomposition of relation modules. J. Pure Appl. Algebra 69 (1990), 135-140. Zbl 0756.20008 MR 1086556

[24] R. Guralnick, T. Penttila, C. E. Praeger, and J. Saxl, Linear groups with orders having certain large prime divisors. Proc. London Math. Soc. (3) 78 (1999), 167-214. Zbl 1041.20035 MR 1658168

[25] R. M. Guralnick and J. Saxl, Generation of finite almost simple groups by conjugates. J. Algebra 268 (2003), 519-571. Zbl 1037.20016 MR 2009321

[26] R. M. Guralnick and P. H. Tiep. Some bounds on $H^{2}$. In preparation.

[27] R. W. Hartley, Determination of the ternary collineation groups whose coefficients lie in the GF $\left(2^{n}\right)$. Ann. of Math. (2) 27 (1925), 140-158. JFM 51.0117.07 MR 1502720

[28] C. Hoffman, On the cohomology of the finite Chevalley groups. J. Algebra 226 (2000), 649-689. Zbl 0959.20017 MR 1752754

[29] D. F. Holt, Exact sequences in cohomology and an application. J. Pure Appl. Algebra 18 (1980), 143-147. Zbl 0439.18016 MR 585219

[30] D. F. Holt, On the second cohomology group of a finite group. Proc. London Math. Soc. (3) 55 (1987), 22-36. Zbl 0624.20035 MR 887282

[31] I. Korchagina and A. Lubotzky, On presentations and second cohomology of some finite simple groups. Publ. Math. Debrecen 69 (2006), 341-352. Zbl 05150123 MR 2273986 
[32] V. Landazuri and G. M. Seitz, On the minimal degrees of projective representations of the finite Chevalley groups. J. Algebra 32 (1974), 418-443. Zbl 0325.20008 MR 0360852

[33] A. Lubotzky, Enumerating boundedly generated finite groups. J. Algebra 238 (2001), 194-199. Zbl 1052.20017 MR 1822189

[34] A. Lubotzky, Pro-finite presentations. J. Algebra 242 (2001), 672-690. Zbl 0985.20017 MR 1848964

[35] A. Lubotzky, Finite presentations of adelic groups, the congruence kernel and cohomology of finite simple groups. Pure Appl. Math. Q. 1 (2005), 241-256. MR 2194724

[36] A. Lubotzky and D. Segal, Subgroup growth. Progr. Math. 212, Birkhäuser, Basel 2003. Zbl 1071.20033 MR 1978431

[37] S. MacLane, Homology. Grundlehren Math. Wiss. 114, Springer-Verlag, Berlin 1963. Zbl 0133.26502 MR 0156879

[38] G. Malle, The maximal subgroups of ${ }^{2} F_{4}\left(q^{2}\right)$. J. Algebra 139 (1991), 52-69. Zbl 0725.20014 MR 1106340

[39] A. Mann, Enumerating finite groups and their defining relations. J. Group Theory 1 (1998), 59-64. Zbl 0892.20018 MR 1490158

[40] H. H. Mitchell, Determination of the ordinary and modular ternary linear groups. Trans. Amer. Math. Soc. 12 (1911), 207-242. JFM 42.0161.01 MR 1500887

[41] P. M. Neumann, An enumeration theorem for finite groups. Quart. J. Math. Oxford Ser. (2) 20 (1969), 395-401. Zbl 0204.34701 MR 0254134

[42] R. M. Pawloski, Computing the cohomology ring and Ext-algebra of group algebras. Ph.D. Thesis, University of Arizona, Tucson 2006.

http://bluehawk.monmouth.edu/ rpawlosk/solutions/dissertation final.pdf

[43] J.-P. Serre, Galois cohomology. Springer Monogr. Math., Springer-Verlag, Berlin 2002. Zbl 1004.12003 MR 1867431

[44] R. Steinberg, Generators, relations and coverings of algebraic groups, II. J. Algebra 71 (1981), 527-543. Zbl 0468.20038 MR 630615

[45] J. G. Sunday, Presentations of the groups SL(2, m) and PSL(2, m). Canad. J. Math. 24 (1972), 1129-1131. Zbl 0253.20051 MR 0311782

[46] M. Suzuki, On a class of doubly transitive groups. Ann. of Math. (2) 75 (1962), 105-145. Zbl 0106.24702 MR 0136646

[47] M. Suzuki, On a class of doubly transitive groups: II. Ann. of Math. (2) 79 (1964), 514-589. Zbl 0123.25101 MR 0162840

[48] G. E. Wall, Some applications of the Eulerian functions of a finite group. J. Austral. Math. Soc. 2 (1961/1962), 35-59. Zbl 0121.27302 MR 0125156

[49] W. Willems, On irreducible faithful modules and their cohomology. Bull. London Math. Soc. 23 (1991), 75-77. MR 1111538

[50] J. S. Wilson, Finite axiomatization of finite soluble groups. J. London Math. Soc. (2) 74 (2006), 566-582. Zbl 05123024 MR 2286433 
[51] R. A. Wilson et al., ATLAS of finite group representations, version 3. http://brauer.maths.qmul.ac.uk/Atlas/v3/

Received December 12, 2006; revised May 6, 2007

R. Guralnick, Department of Mathematics, University of Southern California, Los Angeles, CA 90089-2532, U.S.A.

E-mail: guralnic@usc.edu

W. M. Kantor, Department of Mathematics, University of Oregon, Eugene, OR 97403-1222, U.S.A.

E-mail: kantor@math.uoregon.edu

M. Kassabov, Department of Mathematics, Cornell University, Ithaca, NY 14853, U.S.A. E-mail: kassabov@math.cornell.edu

A. Lubotzky, Department of Mathematics, Hebrew University, Givat Ram, Jerusalem 91904, Israel

E-mail: alexlub@math.huji.ac.il 